\title{
A proposal for the structure of high- and low-density fluctuations in liquid water
}

Cite as: J. Chem. Phys. 151, 034508 (2019); https://doi.org/10.1063/1.5100875

Submitted: 21 April 2019 . Accepted: 24 June 2019 . Published Online: 19 July 2019

Gaia Camisasca (D), Daniel Schlesinger (D), lurii Zhovtobriukh, George Pitsevich (D), and Lars C. M. Pettersson (D)

ARTICLES YOU MAY BE INTERESTED IN

Is water one liquid or two?

The Journal of Chemical Physics 150, 234503 (2019); https://doi.org/10.1063/1.5096460

Ions' motion in water

The Journal of Chemical Physics 150, 190901 (2019); https://doi.org/10.1063/1.5090765

Thermodynamic analysis of the stability of planar interfaces between coexisting phases and its application to supercooled water

The Journal of Chemical Physics 150, 224503 (2019); https://doi.org/10.1063/1.5097591

The Journal

Submit Today

of Chemical Physics

The Emerging Investigators Special Collection and Awards Recognizing the excellent work of early career researchers! 


\title{
A proposal for the structure of high- and low-density fluctuations in liquid water
}

\author{
Cite as: J. Chem. Phys. 151, 034508 (2019); doi: 10.1063/1.5100875 \\ Submitted: 21 April 2019 - Accepted: 24 June 2019 • \\ Published Online: 19 July 2019
}

\author{
Gaia Camisasca, ${ }^{1}$ (D) Daniel Schlesinger, ${ }^{2}$ (D) Iurii Zhovtobriukh, George Pitsevich, \\ and Lars G. M. Pettersson ${ }^{1, a)}$ (D)
}

\begin{abstract}
AFFILIATIONS
'Department of Physics, AlbaNova University Center, Stockholm University, S-106 91 Stockholm, Sweden

${ }^{2}$ Department of Environmental Science and Analytical Chemistry \& Bolin Centre for Climate Research, Stockholm University, 11418 Stockholm, Sweden

${ }^{3}$ Belarusian State University, Nezavisimosti Ave., 4, 220030 Minsk, Belarus
\end{abstract}

Note: This paper is part of a JCP Special Topic on Chemical Physics of Supercooled Water.

a) Author to whom correspondence should be addressed: Igm@fysik.su.se

\begin{abstract}
Based on recent experimental data that can be interpreted as indicating the presence of specific structures in liquid water, we build and optimize two structural models which we compare with the available experimental data. To represent the proposed high-density liquid structures, we use a model consisting of chains of water molecules, and for low-density liquid, we investigate fused dodecahedra as templates for tetrahedral fluctuations. The computed infrared spectra of the models are in very good agreement with the extracted experimental spectra for the two components, while the extracted structures from molecular dynamics (MD) simulations give spectra that are intermediate between the experimentally derived spectra. Computed x-ray absorption and emission spectra as well as the O-O radial distribution functions of the proposed structures are not contradicted by experiment. The stability of the proposed dodecahedral template structures is investigated in MD simulations by seeding the starting structure, and remnants found to persist on an $\sim 30$ ps time scale. We discuss the possible significance of such seeds in simulations and whether they can be viable candidates as templates for structural fluctuations below the compressibility minimum of liquid water.
\end{abstract}

Published under license by AIP Publishing. https://doi.org/10.1063/1.5100875

\section{INTRODUCTION}

Water is fundamental to life and our environment, where most processes take place in the presence of water. It has attracted significant attention due to its importance and the many unresolved questions around the origin of its unusually large number of anomalous properties. ${ }^{1-5}$ Recently, new experimental techniques based on $\mathrm{x}$-ray spectroscopy ${ }^{6}$ and $\mathrm{x}$-ray free-electron lasers, ${ }^{7-10}$ as well as improved theoretical simulation techniques, ${ }^{11,12}$ have led to significant progress in our understanding. The recent data indicate the existence of preferred local structural arrangements in the liquid, which become increasingly well-defined upon supercooling.

However, no details of such structures are presently available.

The proposal that the origin of the anomalous behavior of liquid water could be due to two different forms of the liquid, a high-density liquid (HDL) and a low-density liquid (LDL), is of particular interest in this context. The proposed coexistence line between pure HDL and LDL ending in a critical point ${ }^{16}$ would, however, lie in a highly metastable region of the phase diagram, where rapid crystallization makes experimental studies of the liquid phase extremely challenging. The two different forms would be related to the two amorphous ices, low-density amorphous (LDA) and highdensity amorphous (HDA), between which a first-order-like phase transition in pressure has been observed. ${ }^{17-20}$ Recently, this connection was confirmed by following the transition using wide-angle $\mathrm{x}$-ray scattering (WAXS) as HDA was heated into the ultraviscous regime and simultaneously using $\mathrm{X}$-ray photon correlation spectroscopy (XPCS) in small-angle $\mathrm{x}$-ray scattering (SAXS) geometry to measure the diffusion constant. ${ }^{21}$ Thus, the liquid-liquid transition has been evidenced directly in the ultraviscous regime, and in 
addition, pure LDL was recently reported upon rapid decompression of ice VIII.

The dramatic increase in both compressibility and heat capacity upon supercooling water was shown by Angell and co-workers ${ }^{23-25}$ to be well fitted by a power-law with divergence around $228 \mathrm{~K}$, consistent with a critical point. Recently, Nilsson and co-workers reported SAXS measurements down to $227 \mathrm{~K}$ on micron-sized water droplets falling in a vacuum where a maximum, rather than divergence, was found in the isothermal compressibility. This would be consistent with crossing of the Widom line, i.e., the extension beyond a critical point of the coexistence line into the single-phase region.

There is thus mounting evidence for the liquid water phase diagram being more complex than presumed, with two pure phases in the metastable region leading to structural fluctuations which give rise to the anomalous properties in the stable liquid region. ${ }^{3,26}$ It should be noted that the claim that the liquid-liquid coexistence and transition reported in atomistic simulations s,27-32 $^{16}$ should only be "putative" and rather a liquid-solid transition ${ }^{33,34}$ has now been demonstrated to be incorrect.

Two-state behavior of liquid water is fully consistent with thermodynamics and, in fact, is a requirement for an equation-of-state to reproduce the anomalous behavior of the thermodynamic properties of water. $^{14,37-39}$ It is also consistent with the temperature dependence of the isotropic Raman spectrum of the $\mathrm{OH}$ stretch in $\mathrm{H}_{2} \mathrm{O}$ liquid which exhibits two peaks with an isosbestic point and transfer of intensity between the two. ${ }^{26,40-43}$ The interpretation of isosbestic points as indicating conversion between species has been criticized by Geissler and co-workers, ${ }^{44,45}$ but the criticism does not apply in this case when the integrated intensity is independent of temperature. ${ }^{43}$ Neither does it apply to the infrared (IR) spectrum of $\mathrm{H}_{2} \mathrm{O}$ since Maréchal has shown that the full $\left(0<\tilde{v}<4000 \mathrm{~cm}^{-1}\right)$ temperature-dependent spectrum can be decomposed as the temperature-dependent sum of two temperature-independent spectra; ${ }^{46}$ since the two spectra are fixed and the sum of the mixing coefficients is near constant, then any frequency where the spectra have the same intensity will be a true isosbestic point in the conversion between structural species. A very recent combined experimental and simulation study reported similar conclusions for the full Raman spectrum of liquid water. ${ }^{4}$

Further experimental indications of fluctuations between two local structures are provided by optical Kerr effect measurements of low-frequency modes, ${ }^{48}$ as well as by a wealth of studies using $\mathrm{x}$-ray absorption spectroscopy (XAS) $)^{6,49-55}$ and $\mathrm{x}$-ray emission spectroscopy (XES). ${ }^{56-69}$ This assignment has been questioned and debated, ${ }^{45,70-81}$ where the most recent attempt to reconcile $\mathrm{X}$-ray spectroscopies with a continuous distribution ${ }^{82}$ attracted criticism based on fundamental principles. ${ }^{83}$ Thus, the scales seem to weigh more and more over to the two-state picture. ${ }^{3}$ Indeed, the polarization dependence of the two peaks in the $\mathrm{H}_{2} \mathrm{O}$ Raman spectrum was used by Scherer et al. ${ }^{84}$ already in 1974 to assign the low-energy peak to tetrahedral symmetric structures and the high-energy peak to disordered local structures in a ratio of 1:3. A similar assignment in terms of structures was 30 years later given by Wernet et al. ${ }^{52}$ based on XAS, and a similar ratio (1:2.5 \pm 0.5$)$ has also been obtained from XES.

Additional information on the structure of liquid water is obtained from recent high-precision measurements of the x-ray scattering structure factor out to very large Q-transfer $\left(\sim 26 \AA^{-1}\right)^{86,87}$ allowing us to determine a benchmark $\mathrm{O}-\mathrm{O}$ radial distribution function (RDF) at ambient conditions. ${ }^{87}$ Furthermore, temperaturedependent measurements showed peak structure out to $\sim 17 \AA$ with peak heights that varied, with some decreasing and some increasing, when lowering the temperature toward supercooled conditions; ${ }^{88}$ in particular, the connection between the minimum in isothermal compressibility and structural changes in terms of increased tetrahedrality has been pointed out. ${ }^{86}$ The temperature dependence of the peaks at the intermediate and long range was analyzed by Schlesinger et al. ${ }^{88}$ in terms of a changing balance between HDL and LDL local environments in the liquid. This was further elaborated and connected to other experimental observations in a recent review, ${ }^{26}$ and, in an accompanying study ${ }^{13}$ in the present volume, the peaks at the intermediate range in the $\mathrm{O}-\mathrm{O}$ pair-distribution are shown to become very sharp and well-defined upon supercooling down to $235 \mathrm{~K}$. However, apart from general statements, e.g., icelike and something else by Röntgen, ${ }^{89}$ the proposed chains or rings vs tetrahedral by Wernet et al., ${ }^{52}$ and tetrahedral vs disordered by Scherer et al., ${ }^{84}$ no specific structures for the tetrahedral component in the size range of $\sim 10$ Å radius as found experimentally ${ }^{60,88,90}$ have been established so far. However, we note the early proposal of clathrate structures by Pauling ${ }^{91}$ and the observation by Walrafen ${ }^{92}$ that the Raman spectrum of clathrates is very close to that of supercooled water and the suggestion of clathrate structures by Yokoyama et al..$^{93}$ from x-ray diffraction (XRD).

In the present work, we investigate the hydrogen bond topology in terms of minimal ring-structures in water, simulated using the TIP4P/2005 force-field, ${ }^{94}$ and note that pentagonal rings dominate in the simulation when the temperature is decreased as already discussed by Speedy in $1984 .{ }^{95}$ The preference for pentagonal rings has been noted by Santra et al. ${ }^{96}$ for the LDL component in simulated ambient water, and a discussion of pentamers in liquid water was recently published by Mandziuk, ${ }^{97}$ although a recent study by Martelli ${ }^{98}$ found significant contributions also from hexagons. Tanaka has suggested that the preference for pentagonal, rather than hexagonal, rings can frustrate ice nucleation. ${ }^{99}$ We thus build model structures using fused dodecahedra containing only pentagons to represent LDL and using chains of water molecules to represent HDL, as suggested by Wernet et al. ${ }^{52}$ These are then fully optimized based on density functional theory (DFT) including van der Waals interactions, and the full infrared spectrum is computed for each model. These spectra are then compared with the extracted temperature-independent spectra from Maréchal. ${ }^{46}$

The two constructed models are found to give spectra in close agreement with the spectra from Maréchal, while a sampling of structures taken from the simulation to represent either HDL or LDL local environments does not. We furthermore compare the total energy of the 103-molecule clathratelike dodecahedral cluster with a sampling of similarly DFT optimized 103-molecule clusters taken from a simulation using the TIP4P/2005 force-field, and among these structures, as well as for a 103-molecule ice Ih cluster, the clathratelike is lowest in energy. We do not expect that these specific structures are likely to spontaneously appear in any finitelength, finite box size simulation of water and instead seed simulations with such structures and investigate how long they can persist. We propose that clathratelike structures, similar to the here studied 
fused dodecahedra, can represent the LDL local structures in real water as templates around which fluctuations occur, while chains can represent the HDL-like structures.

\section{METHODS}

\section{Models}

The chain model (Fig. 1a) was based on the NE/S/E/SW structure model of high-density solid water (HSW) of Anick. ${ }^{100}$ This has a layered structure with $\mathrm{H}$-bonded chains of molecules, where the compass-based acronym specifies the directionality of $\mathrm{H}$-bonding in and between layers. ${ }^{100}$ From this structure, nine chains of eight molecules each were extracted, with the central chain fully embedded by the neighboring chains. The density $\left(1.40 \mathrm{~g} / \mathrm{cm}^{3}\right)$ of the original HSW model was adjusted to $\sim 1.1 \mathrm{~g} / \mathrm{cm}^{3}$ to mimic an expected density difference between HDL and LDL of $\sim 20 \%$. In the optimization, the oxygen in molecules terminating each end of the chains was fixed to avoid collapse of the model.

The model of LDL has a central dodecahedron to which seven additional dodecahedra were fused to generate a cluster consisting of a total of 103 molecules, which was fully geometry optimized (Figs. 1b and 1c). To compare with local structures extracted from a molecular dynamics (MD) simulation, we used an NVT simulation of ambient TIP4P/2005 water ${ }^{94}$ from which we selected four

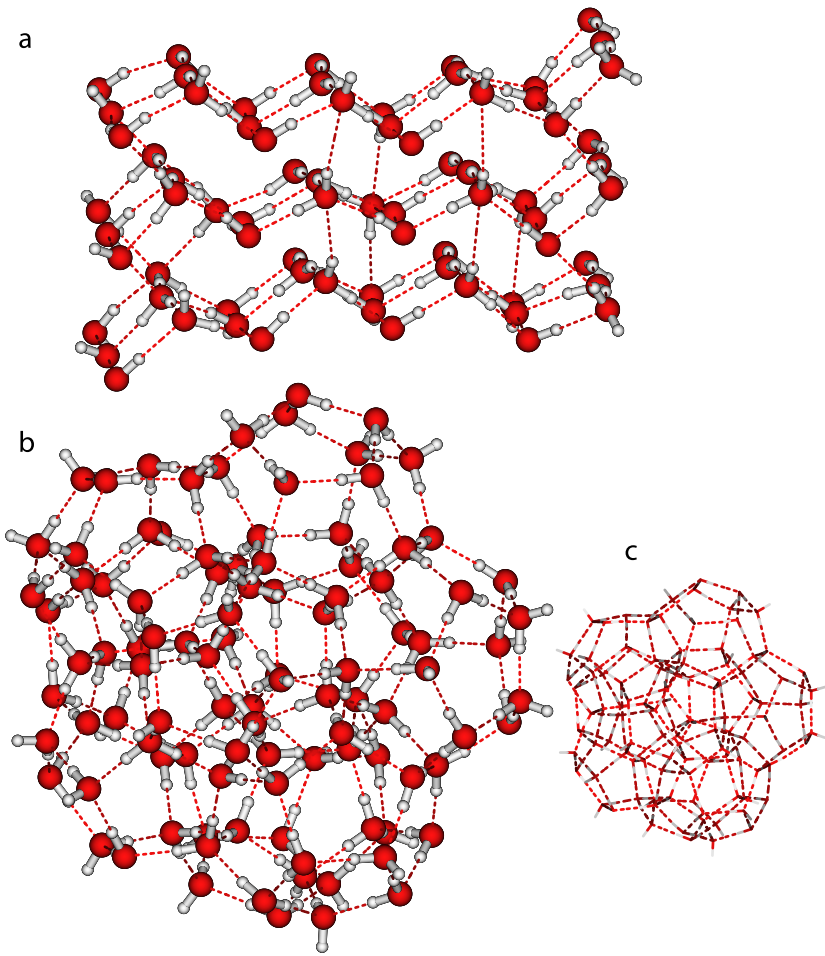

FIG. 1. (a) Optimized chain model. The oxygens terminating each chain have been fixed in position during the optimization, while all other atoms were free to move. (b) Optimized structure of fused dodecahedra with (c) the outline of the structure for better visualization. 103-molecule structures based on the local-structure index (LSI) ${ }^{101}$ characterization in the real structure (i.e., including temperature) as either strongly HDL-like (LSI $<0.002 \AA^{2}$ ) or strongly LDL-like (LSI > $0.20 \AA^{2}$ ). The LSI order parameter measures the degree of order out to the second hydration shell in the O-O pair-distribution function. The same number of molecules as for the fused dodecahedra was used in order to allow a comparison of total interaction energies. The clusters were built using the 102 nearest neighbors to the selected molecule that fulfilled the LSI criterion in the snapshot. In addition, we extracted from the simulation three additional structures, where not only the central molecule was classified as HDL or LDL according to the LSI criterion but also the immediate neighbors.

\section{Structure optimization and infrared spectra}

The geometry optimizations and infrared spectrum calculations were performed using Gaussian $16^{102}$ with the $6-311 \mathrm{G}^{*}$ basis set and the wB97XD range-separated functional, ${ }^{103}$ which includes an estimate of dispersion. The models were furthermore embedded in a polarizable medium using the SMD reaction field $^{104}$ to reduce edge effects and better mimic potential structures in the liquid. The optimized structures of the model chains and fused dodecahedra are shown in Fig. 1, and coordinates are given in the supplementary material. To compare with hexagonal ice Ih, a cluster of 103 molecules was furthermore extracted from a perfect ice lattice and optimized using the same procedure including embedding in polarizable medium. To maintain the ice structure of the cluster, it was, however, found necessary to fix the oxygen in 25 of the molecules terminating the structure.

\section{X-ray spectroscopy calculations}

XES and XAS spectra were computed for the chain and dodecahedral models including all 72 water molecules in the case of the chain model and 103 water molecules in the case of the dodecahedral structure. Both for the chains and dodecahedra, the spectrum contributions were computed only for the central, fully coordinated molecules, i.e., six molecules for the chains and eight for the dodecahedra.

The XAS spectra have been obtained using a complex polarization propagator in combination with Kohn-Sham DFT electronic structure theory $(\mathrm{CPP}-\mathrm{DFT})^{105,106}$ as implemented in the Dalton code. $^{107}$ The used exchange-correlation functional was CAMB3LYP $^{108}$ with modified parameters $(\mu=0.33, \alpha=0.19$, and $\beta=0.81)$ which ensures correct asymptotic behavior of the long-range Coulomb interaction between the excited electron and remaining positive molecular core. These functional parameters have demonstrated good accuracy in reproducing the XAS spectrum profile for a series of small molecules. ${ }^{109,110}$ The oxygen in the excited water molecule was described with the IGLO-III $[7 s 6 p 2 d]^{111}$ basis set, while for the hydrogens (including the six nearest neighbor molecules) the IGLO-II $[3 s 1 p]^{111}$ basis was employed. The remaining oxygens were described via an effective core potential (MWB) $)^{112}$ in order to uniquely define the core-excited molecule. The oxygens in the six closest neighbor molecules were described by a 
triple- $\zeta[3 s 3 p 1 d]$ basis set obtained from the corresponding double- $\zeta$ MWB basis set ${ }^{112}$ by uncontracting the third primitive Gaussian function in the first contracted Gaussian orbital and adding a $d$-function with exponent 0.7 . For the remaining molecules, a double- $\zeta[2 s]$ basis set ${ }^{113}$ was used to describe hydrogens and the MWB [2s2p] double- $\zeta$ basis set ${ }^{112}$ with the last $p$-function removed was employed for oxygens; using smaller basis sets on the surrounding molecules reduces the computational cost significantly without affecting the accuracy of the spectrum simulations. ${ }^{114}$ In order to improve the spatial sampling of extended excited states, the basis set on the core-excited water molecule was extended by adding the $19 s 19 p 19 d$ augmentation basis set ${ }^{115}$ containing very diffuse $s, p$, and $d$ exponents. A common life-time broadening parameter $\gamma=1000 \mathrm{~cm}^{-1}(0.124 \mathrm{eV})$ has been utilized for all excited states. The calculated excitation energies are too low in comparison with the experiment due to the approximate exchange-correlation functional resulting in self-interaction error. Due to this, the computed XAS spectra were shifted using a constant shift around $15 \mathrm{eV}$ in order to fit the experimental pre-edge position.

The XES spectra have been calculated using time-dependent density functional theory (TD-DFT) ${ }^{116}$ within the Tamm-Dancoff approximation, ${ }^{117}$ as implemented in the Q-CHEM code. ${ }^{118}$ The excitation energies and oscillator strengths were computed as deexcitations from valence states into a core hole at the selected $1 s$ oxygen orbital. The oscillator strengths were convoluted with a Lorentzian with $0.18 \mathrm{eV}$ full width at half maximum to obtain a continuous spectrum. The $\mathrm{B}^{\mathrm{X}} \mathrm{LYP}$ functional with $\mathrm{X}=0.66$ giving the fraction of Hartree-Fock exchange was used which has been specially optimized for XES calculations for second row elements. ${ }^{119}$ The water molecule for which XES was computed was described by the IGLO-III basis, ${ }^{111}$ which is specially designed to improve the core orbital description. The remaining molecules were described using the Pople 6-31G basis set. ${ }^{120}$

\section{Molecular dynamics simulations}

To investigate the stability of the dodecahedral clusters and the effect of temperature on their radial distribution functions, gas phase MD simulations were performed using the GROningen MAchine for Chemical Simulations (GROMACS) ${ }^{121}$ and the TIP4P/2005 forcefield. ${ }^{94}$ The simulations were performed with a time step of $1 \mathrm{fs}$ and in double-precision mode to improve conservation of energy which reduced the drift to $0.0008(\mathrm{~kJ} / \mathrm{mol}) / \mathrm{ps}$. Additional clusters of 119 and 142 molecules were constructed by adding more dodecahedra in order to extend the $\mathrm{O}-\mathrm{O}$ pair-distribution function to longer distances to compare with the experiment on the liquid. The structures were first energy-minimized to obtain the initial bond-distances relevant for the force-field. Gas phase simulations were performed for the dodecahedral clusters of 119 and 142 molecules using an initial energy minimization at $0 \mathrm{~K}$. This was followed by NVT simulations at 100 and $200 \mathrm{~K}$ for 200 ps followed by 20 ps in the NVE ensemble which is enough to obtain a converged RDF.

To investigate the stability of the dodecahedral structure in simulations of water, we solvated the dodecahedral cluster of 142 TIP4P/2005 molecules into TIP4P/2005 water. The dodecahedral cluster was placed in the center of a cubic box with $10.765 \mathrm{~nm}$ side. This also ensures that the cluster does not interact with its periodic images. Then, we filled the box with water molecules using a GROMACS standard procedure, which also removes overlapping water molecules based on the atoms' van der Waals radii. The final density of this system, containing 40988 solvent water molecules plus the 142 composing the dodecahedra, is $985.2 \mathrm{~kg} / \mathrm{m}^{3}$. For the solvated dodecahedral cluster, we first equilibrate the solvent around the cluster. This is done in the NPT ensemble by applying a positionrestraining force on the atoms of the cluster, for $1.7 \mathrm{~ns}$ at $250 \mathrm{~K}$ and 1 bar. The position restraint allows us to equilibrate the solvent water around the dodecahedral cluster without causing structural changes on it during this step, unless an energy penalty is paid. The equilibration of the solvent is monitored through the convergence of the potential energy toward a steady value which was $51.13 \mathrm{~kJ} / \mathrm{mol}$ per molecule in agreement with the $51.129 \mathrm{~kJ} / \mathrm{mol}$ per molecule in a bulk simulation of 45000 molecules at the same thermodynamic conditions. ${ }^{122}$ The temperature was controlled by the velocity-rescaling algorithm $^{123}$ with a time constant of 0.01 ps and the pressure by the Berendsen method ${ }^{124}$ with a time constant of 0.2 ps. The output configuration, with a box side of $10.734 \mathrm{~nm}$, was used to run an NVT simulation at $250 \mathrm{~K}$ for 50 ps to follow the rearrangement of the cluster (i.e., without position restraint). We decided to perform this run in the NVT ensemble because under NPT conditions the coordinates of the template cluster would be directly rescaled, which would in turn lead to perturbation of its structure due to the external influence of the barostat.

\section{Topological ring analysis}

A graph-theoretical characterization of TIP4P/2005 water was performed with an implementation of a recursive ring-search algorithm to determine the ring-statistics in the simulated liquid, i.e., the shortest closed loops leading back to the selected $\mathrm{H}$-bond donor molecule. The hydrogen-bond (H-bond) adjacency matrix, where the H-bonds are defined using the cone criterion of Wernet et al., ${ }^{52}$ or alternatively a $3.5 \AA / 30^{\circ}$ criterion, was established for frames from molecular dynamics simulations of $\mathrm{N}=512 \mathrm{TIP} 4 \mathrm{P} / 2005$ molecules at a pressure of $\mathrm{P}=1 \mathrm{bar}$ and temperatures of $\mathrm{T}=200-350 \mathrm{~K}$ in steps of $\Delta \mathrm{T}=30 \mathrm{~K}$ and of proton-disordered hexagonal ice at $\mathrm{T}=70 \mathrm{~K}$. The simulations were run for $1 \mu \mathrm{s}$ for $\mathrm{T}=200-230 \mathrm{~K}$ and $500 \mathrm{~ns}$ for $\mathrm{T}=240-370 \mathrm{~K}$. This is more than sufficient to converge structural properties, although dynamical properties below the Widom line (230 K for TIP4P/2005) require longer simulations to converge. $^{37}$

Starting from an initial molecule, an H-bonded neighbor is identified from the adjacency matrix. The algorithm then tests this neighbor for $\mathrm{H}$-bonds connecting back to the initial molecule, excluding the latest $\mathrm{H}$-bond instance. This simple scheme is called recursively until either a ring is closed or a given maximum iteration depth is reached, typically ten iterations. The same characterization in terms of rings was performed on the dodecahedra solvated in water along the NVT run. Configurations of 350-450 molecules composed by the dodecahedra and the solvation water molecules within $0.4 \mathrm{~nm}$ from the dodecahedra were selected along the NVT run for $\mathrm{t}=0,5,10,20,30,40$, and 50 ps. For a given time, the ring-distribution has been averaged over 10 frames spaced by $100 \mathrm{fs}$, i.e., $0-0.9$ ps, $4.5-5.5$ ps, $9.5-10.5$ ps, ..., 49.1-50 ps. For reference, 10 bulk configurations of 375 water molecules simulated at similar conditions were also analyzed. 


\section{RESULTS}

\section{H-bond topology}

Figure 2 shows the normalized histograms of smallest ring sizes found in the simulation of equilibrated TIP4P/2005 water as a function of temperature. Evidently, the five-ring frequency, rather than hexagons, is most prominent in the simulated liquid and becomes even more pronounced upon supercooling, while the distribution becomes narrower. This result is not unexpected considering the difficulty to nucleate hexagonal ice in simulations. For ice Ih, the smallest rings that molecules are part of are exclusively six-membered rings, as expected. The result is robust against changes in the $\mathrm{H}$-bond definition. This forms the basis for exploring more extended structural models based on pentagonal subunits and determining whether such models can be excluded based on existing experimental data.

We note that a constraint is that the constructed structures must be distinct from templates that can nucleate space-filling growth, since such structures in the liquid could otherwise act as seeds for crystallization below $0{ }^{\circ} \mathrm{C}$, which would be inconsistent with deep supercooling of water. The strictly pentagonal, fused dodecahedra modeled here fulfill this criterion since, as the structure is extended, more and more angular strain is built up preventing further growth.

\section{Computed infrared spectra}

In Fig. 3, we show the computed IR spectra of the chains and fused dodecahedra in comparison with the HDL and LDL spectra extracted from the experiment by Maréchal. ${ }^{46}$ We justify this way of computing IR spectra from optimized structures by the temperature independence of the two, experimentally extracted, spectra.

Focusing on the $\mathrm{OH}$-stretch region $\left(3000-3700 \mathrm{~cm}^{-1}\right)$, the experimentally extracted LDL and HDL spectra show clear differences with a significantly higher cross section for the tetrahedral LDL component, together with a red-shift reflecting the enhanced

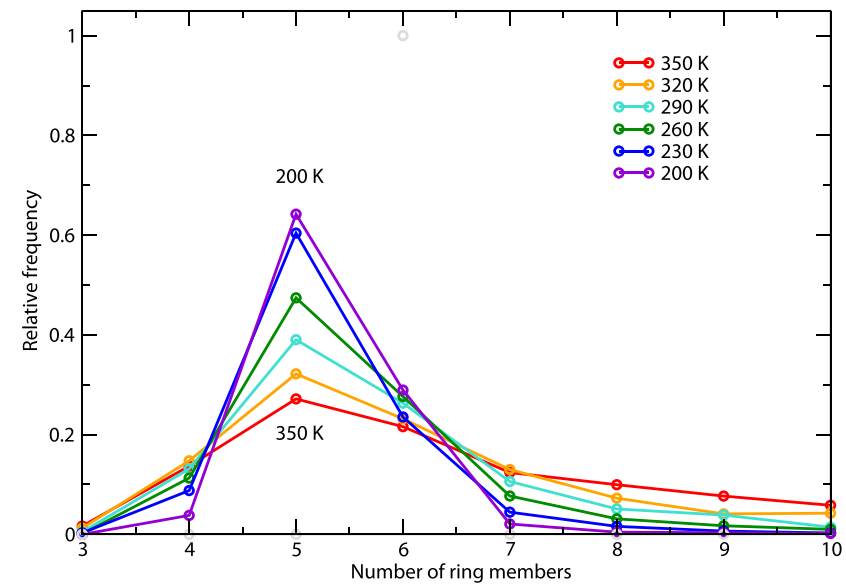

FIG. 2. Relative occurrence of shortest loops as a function of temperature in simulations of TIP4P/2005 water. Pentagonal subunits dominate at all temperatures but become more enhanced at supercooled conditions.

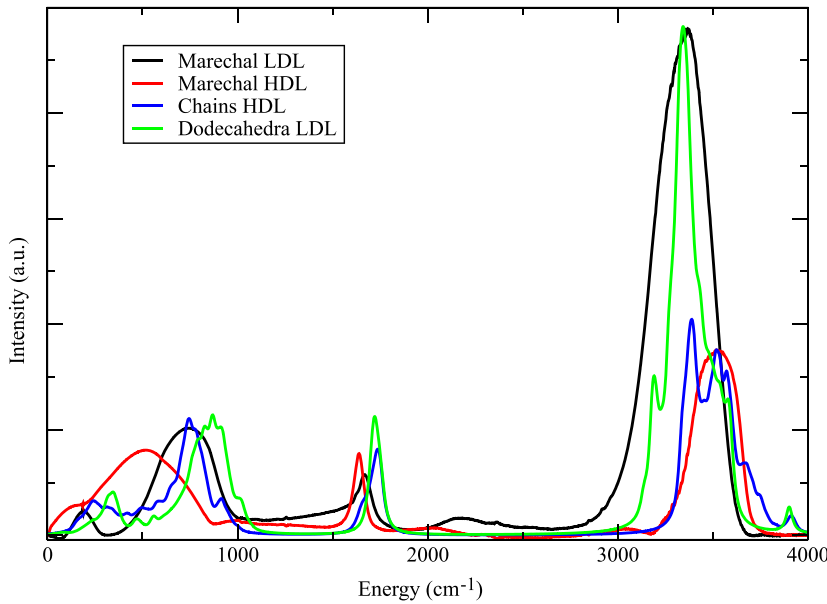

FIG. 3. Comparison of computed IR spectra for chain model (chains HDL, blue) and fused dodecahedra (dodecahedra LDL, green) with spectra extracted from the experiment for HDL (red) and LDL (black) from Ref. 46.

$\mathrm{H}$-bonding. This is very well reflected by the models, where the $\mathrm{OH}-$ stretch spectrum of the fused dodecahedra closely follows the experimentally derived LDL spectrum and the chain-model that of the HDL with significantly lower intensity and an overall blue-shift. The spectrum comparison is limited in the sense that the temperature of the optimized structures corresponds to $0 \mathrm{~K}$ and only one specific structure, albeit with 103 and 72 molecules for the fused dodecahedra and chains, respectively. We conclude for the $\mathrm{OH}$-stretch that the two models are not inconsistent with the experimentally derived spectra.

For the bend around $1600 \mathrm{~cm}^{-1}$, we find that the models give a blue-shift compared to the experiment and also that the intensity relationship is reversed; where the experimentally derived spectra have a slightly higher intensity for the HDL contribution, we instead find a slightly higher intensity for the fused dodecahedra (LDL model). The combination bands around $2000-2200 \mathrm{~cm}^{-1}$ is naturally absent in the computed spectra, which are obtained within the harmonic approximation and thus do not include coupling between modes. A closer analysis of this region in the spectra extracted by Maréchal shows enhanced intensity in the combination band for the extracted LDL spectrum than for HDL which indicates a stronger anharmonic coupling between the bend and librational modes in the case of LDL. This thus provides a plausible explanation of the reversed intensity ratio for the bend since intensity transfer from the bend to the combination band is not included in our spectrum calculation.

Turning to the librational $\left(500-1000 \mathrm{~cm}^{-1}\right)$ and lower-energy collective $\mathrm{H}$-bond-related modes, these are known to be not wellrepresented in a harmonic approximation, and thus only a qualitative comparison between computed and experimentally derived spectra can be made in this region. However, we note that the shape and relative positions of the contributions from the models exhibit the trends of the experimentally derived spectra. Thus, the librational peak of the fused dodecahedra is found at higher energy than for the chain model, although the shift is significantly smaller than in 
the experiment. We note further that the dodecahedral model shows a well-defined peak around $300 \mathrm{~cm}^{-1}$ which corresponds to the experiment for LDL around $200 \mathrm{~cm}^{-1}$. In qualitative agreement with the experiment, the chain model exhibits a peak at lower frequency than for the dodecahedral model, albeit blue-shifted compared to the experiment.

We argue that at ambient conditions these very low-lying modes $\left(20-200 \mathrm{~cm}^{-1}\right)$ will be highly excited and contribute to a softening of the $\mathrm{H}$-bond network, which should contribute to bringing down the frequencies that are below $1000 \mathrm{~cm}^{-1}$ in the models, if temperature were included. The most significant conclusion from the present comparison is thus the close, semiquantitative agreement for the $\mathrm{OH}$-stretch between the models and the experimentally derived spectra, while for the low-energy region we note a qualitative agreement in terms of the shapes and relative shifts of the spectra.

An important question is how these model structures relate to structures obtained in simulations of the liquid. If one selects structures from the simulation based on their local structures, characterized as either strongly HDL or strongly LDL (see the section titled Methods) using the LSI value for the central molecule, will they then conform to the spectra of the models above? Here we use TIP4P/2005 force-field ${ }^{94}$ MD simulations at ambient conditions and extract two structures for each of strongly HDL and strongly LDL based on the central molecule. These structures were then optimized at the DFT level as described in the section title Methods, and the obtained spectra are shown in Fig. 4 in comparison with the experimentally derived spectra.

It is clear that, despite widely different LSI values for the central molecule in the temperature-dependent instantaneous structure, i.e., LSI $<0.002 \AA^{2}$ for HDL and LSI $>0.20 \AA^{2}$ for LDL, they all give very similar spectra when optimized which is consistent with earlier suggestions $^{26,85,125}$ that the structural distribution in MD simulations corresponds to an average between the two extremes suggested by experiment ${ }^{3,26,43,47,60,84,92,126}$ and by thermodynamics. ${ }^{14,15,37-39,127}$

Focusing on the $\mathrm{OH}$-stretch peak, the resulting spectrum seems intermediate between the experimentally extracted contributions. The remaining features (bend, librations, and low-frequency modes) of the computed spectra all collapse onto very similar features and intensities which motivated using the same color blue for the two HDL and green for the two LDL structures. It should be noted that both original HDL structures remained as HDL $\left(\right.$ LSI $\left.<0.02 \AA^{2}\right)$ after optimization, while one of the LDL structures became more HDLlike (LSI $0.05 \AA^{2}$ ) and the other remained as well-defined LDL (LSI $\left.0.28 \AA^{2}\right)$.

We next sample three additional structures from the simulation, where not only the central molecule but also its four nearest neighbors fulfills the LSI criterion as being either HDL or LDLlike. Also in this case, the spectra collapse onto each other and onto the spectra in Fig. 4 (see the supplementary material), thus representing more of an average between the experimentally extracted temperature-independent spectra. This is consistent with, e.g., the simulated Raman $\mathrm{OH}$-stretch spectrum using the MB-pol forcefield, ${ }^{128,129}$ which reproduces the shift toward lower frequency with decreasing temperature, but lacks the bimodality observed experimentally. ${ }^{130}$ Also from x-ray emission spectra computed based on snapshots from simulations, it has been proposed that the obtained simulated structure represents a structural average of the fluctuating species in real water.

\section{X-ray spectroscopy}

In Fig. 5, we show the nonresonant XES spectra computed for the central molecules in the optimized chains and the 103-molecule dodecahedral structure. For the chains, we have also included the spectrum computed for the structure simulated using TIP4P/2005

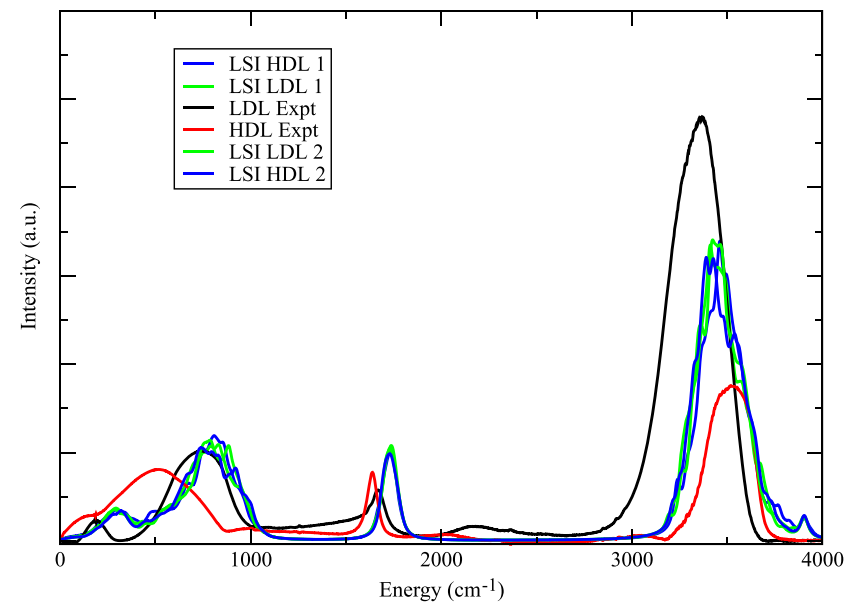

FIG. 4. Computed IR spectra for four optimized 103 molecule structures extracted from a TIP4P/2005 MD simulation. The selection was based on the LSI value of the central molecule as either very disordered (LSI HDL, blue) or very tetrahedral (LSI LDL, green). The spectra extracted from the experiment for HDL (red) and LDL (black) from Ref. 46 are included as a reference.

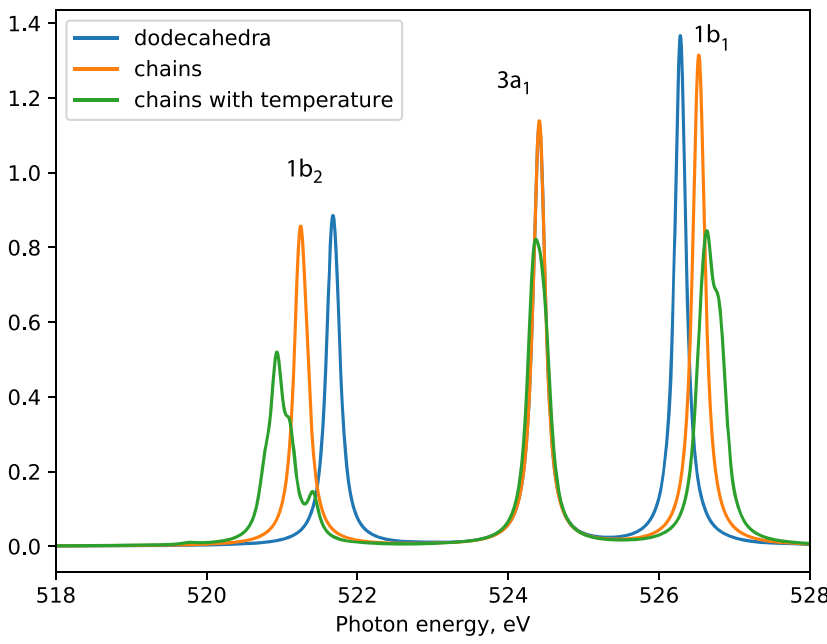

FIG. 5. Computed XES spectra for fully coordinated molecules in the dodecahedral cluster (blue) and chain model with (green) and without (orange) effects of temperature. The computed spectra were area-normalized between 515 and $530 \mathrm{eV}$. 
at $200 \mathrm{~K}$ to include some disorder due to temperature; as when optimizing the structure, the terminating oxygens in the chains were kept fixed.

The spectral features are sharp due to the neglect of vibrational interference effects ${ }^{131,132}$ or core-hole-induced dynamics ${ }^{133}$ due to proton delocalization along the $\mathrm{H}$-bonds during the $\sim 4 \mathrm{fs}^{134}$ core-hole life-time. In earlier studies, ${ }^{135,136}$ we have shown that the peak positions in the $1 b_{1}$ lone-pair region, already without including these effects, are representative of the peak positions with these effects included, although the shape of the peaks will be affected by the dynamical effects. We note that the bonding $1 b_{2}$ and $3 a_{1}$ orbitals in the experiment become strongly broadened due to the dynamics, but the nonbonding $1 b_{1}$ lone-pair remains sharp. ${ }^{56,61,62}$ In liquid water, the lone-pair region exhibits two sharp peaks with temperature-dependent relative intensity ${ }^{56,60-62,133}$ where the peak at lower emission energy has been assigned to tetrahedral (LDL) structures and the peak at higher emission energy to disordered (HDL) structures. ${ }^{53,60-62,125,133}$ The chains (HDL) and dodecahedral (LDL) models considered here are consistent with this assignment, with the chains giving the $1 b_{1}$ peak at higher emission energy and the dodecahedra giving the computed lower emission energy. A direct comparison with the experiment would require a significantly bigger sampling of structures within these structural classes and inclusion of dynamical effects, which at present becomes prohibitive for these size structures. We thus limit the discussion to a qualitative comparison, but note that including temperature in the chains model (Fig. 5) broadens the $1 b_{1}$ peak and gives a shift toward higher energy which is consistent with the experiment and indicative of a looser $\mathrm{HB}$ network when the liquid is heated. Alternative interpretations of the experimental spectra in terms of one peak due to dissociated and the other to intact molecules ${ }^{56}$ or one peak of $3 a_{1}$ symmetry and the other $1 b_{1}{ }^{82,137-139}$ have been disproven experimentally by direct measurements of the contribution from dissociation ${ }^{14}$ and by measurements of the orbital symmetry of the two lone-pair peaks. $^{63,64}$

In Fig. 6, we show computed XAS for the two models in comparison with the experimental benchmark XAS spectrum obtained in transmission by Nilsson and co-workers. ${ }^{49}$ There are three spectral regions of interest which are the sharp pre-edge around $535 \mathrm{eV}$, the main-edge close to $537 \mathrm{eV}$, and the broad postedge at 541$542 \mathrm{eV}$. Upon heating the liquid, the intensity at the pre- and mainedge increases, consistent with assigning these features to broken or weakened H-bonds. ${ }^{52}$ On the other hand, the intensity at the postedge decreases when the liquid is heated, which is consistent with the loss of the tetrahedral structure, since the postedge dominates in XAS of hexagonal ice.

The computed spectra of the chain and dodecahedral models are qualitatively consistent with this assignment with a higher preedge intensity for the chain model, particularly when temperature is included, although in comparison with the experiment the intensity is strongly underestimated. The dodecahedral model gives a dominant contribution at the proper position in the postedge region where the inclusion of a sampling of the $\mathrm{O}-\mathrm{H}$ stretch distribution (not included here) has been shown to significantly broaden the sharp postedge peak. ${ }^{141,142}$ Both models contribute to the main-edge where we note that the chain model contribution is enhanced when thermal disorder is included. Due to the high computational cost in computing XAS on the 103-molecules cluster, it was not feasible

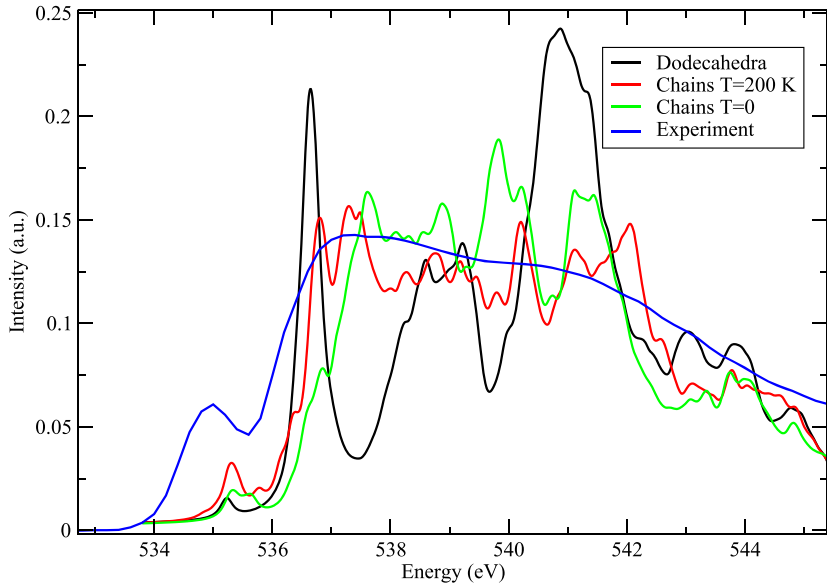

FIG. 6. Computed XAS spectra for fully coordinated molecules in the dodecahedra cluster (black) and chains with (red) and without (green) temperature compared with the experimental XAS spectrum of ambient liquid water measured in transmission from Ref. 49. Computed XAS spectra were area normalized between 535 and $546 \mathrm{eV}$ and scaled for comparison with the experiment.

to directly investigate the effect of temperature on the spectra of the fused dodecahedra. However, introducing disorder through temperature can be expected to significantly broaden the sharp main-edge and further broaden also the postedge in the computed spectrum. Here, it should also be noted that including the quantum mechanical $\mathrm{O}-\mathrm{H}$ distance probability distribution has been shown to be important for obtaining the correct width of spectra of hexagonal ice. $^{141,142}$

We conclude that the two structural models cannot be excluded from consideration as structures in real water based on the computed XES and XAS spectra, although to represent the spectrum of the liquid a significantly larger sampling should be performed and, in particular, including more disordered structures which give greater contributions to the pre-edge. The temperature, $200 \mathrm{~K}$, used here for the chains is below the Widom line, $230 \mathrm{~K}$, of the TIP4P/2005 model and thus corresponds to very deeply supercooled water which enhances $\mathrm{H}$-bond interactions that quench the pre-edge intensity. ${ }^{52,53,55,143}$ The simulation of the chains was done in a vacuum and using the TIP4P/2005 force-field, which necessitated using this very low temperature to avoid evaporation.

\section{O-O radial distribution function}

In Fig. 7, we compare the O-O RDF for liquid water and the dodecahedral clusters simulated using TIP4P/2005 in order to include temperature. The simulations were done for two sizes of clusters, 119 and 142 molecules, in order to extend the RDF to intermediate-range distances that in the experimental RDF show peaks that become more prominent and well-defined at lower temperatures. ${ }^{13,88}$ These are, in particular, the second shell at $\sim 4.5 \AA$, the fourth at $\sim 8.5 \AA$ and the fifth at $\sim 10.7 \AA .{ }^{13,88}$ These can be associated with the LDL component in the liquid which becomes more abundant and structurally well-defined at lower temperatures, providing specific peaks on the less-structured background of more disordered 


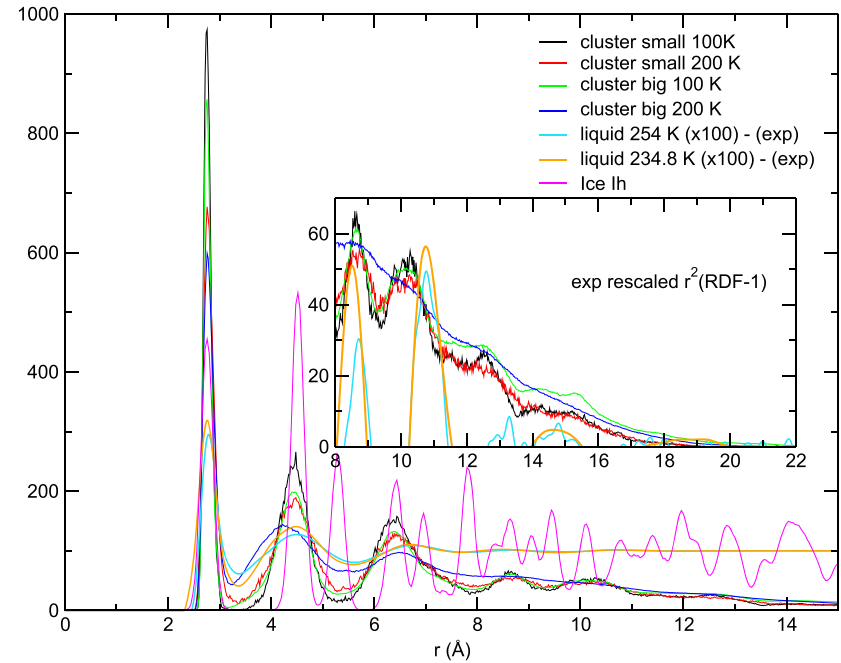

FIG. 7. Comparison of O-O distance distributions in liquid water at $254 \mathrm{~K}$ (light blue) from Ref. 86 , at $234.8 \mathrm{~K}$ (orange) from Ref. 13, hexagonal ice (magenta), and 119 (cluster small) and 142 (cluster big) molecule dodecahedral clusters at 100 and $200 \mathrm{~K}$ simulated using the TIP4P/2005 force-field

HDL contributions to the RDF. In Fig. 7, we include a comparison with the peak positions in hexagonal ice Ih which clearly shows that possible hexagonal, icelike local structures in the liquid must be limited in size to at most the second shell, i.e., $4.5 \AA$ since the hexagonal structure gives peaks at $\sim 5.2 \AA$ and around $8 \AA$ where the measured liquid water $\mathrm{O}-\mathrm{O} \mathrm{RDF}$ exhibits minima. Thus, this motivates the search for alternative structural templates, such as the here studied dodecahedral clusters.
The peaks that, based on the behavior as a function of temperature, can be assigned to LDL-like local structures in the liquid are the second, fourth, and fifth shells for which the dodecahedral models show a good representation in terms of peak positions. In particular, the second and fourth shells are in excellent agreement with the experiment, although the latest data obtained for water at $234.8 \mathrm{~K}^{13}$ indicate a slightly shorter distance for the fourth peak than obtained in the present simulations based on the dodecahedral clusters. The fifth peak in the simulations of the dodecahedral clusters is found at $\sim 0.5 \AA$ shorter distance than the experiment. However, it should be noted that these simulations were performed for the isolated clusters in the gas phase and molecules at these separations will be close to the edges of the cluster which may lead to contracted distances.

\section{Energetics}

A more stringent test of the hypothesis of specific template structures in the liquid will be provided by investigating their persistence and stability as solvated in an aqueous environment. In Table I, we compare the stability of the 103-molecule dodecahedral structure with that of the four similarly optimized 103-molecule clusters with the central molecule in the LDL and HDL environment extracted from the TIP4P/2005 simulations; the other three structures which are even more stringently HDL or LDL in terms of also the nearest neighbors falling in the same category give very similar results.

We find that the dodecahedral structure is the lowest in energy at all levels of calculation presented in Table I. All structures were optimized at the wB97XD ab initio DFT level embedded in a polarizable medium as described in the section titled Methods. For comparison with the TIP4P/2005 and MB-pol results (columns 1 and 2), we also report the wB97XD energetics computed in the optimized geometry but without the polarizable medium in column 3 .

TABLE I. Computed relative energetics of the clusters with 103 molecules as fused dodecahedra and the two LDL and two HDL structures from Fig. 4 and a model of hexagonal ice. Energetics are also obtained using the TIP4P/2005 and MB-pol force-fields without polarizable medium for the wB97XD optimized structures. As comparison, the wB97XD single-point energy without SMD embedding is given together with the total dipole moment of each structure (in italics, Debye). wB97XD gives the relative energies computed with the wB97XD functional and SMD embedding. The additional columns give energies with corrections as indicated. All energies are in eV.

\begin{tabular}{|c|c|c|c|c|c|c|c|}
\hline Structure & $\begin{array}{c}\text { TIP4P/2005 } \\
(\mathrm{eV})\end{array}$ & $\begin{array}{c}\text { MBPol } \\
(\mathrm{eV})\end{array}$ & $\begin{array}{l}\text { wB97XD No } \\
\text { SMD (eV/D) }\end{array}$ & $\begin{array}{c}\text { wB97XD } \\
(\mathrm{eV})\end{array}$ & $\begin{array}{c}\text { Sum of electronic } \\
\text { and zero-point } \\
\text { energies }(\mathrm{eV})\end{array}$ & $\begin{array}{c}\text { Sum of electronic } \\
\text { and thermal } \\
\text { energies }(\mathrm{eV})\end{array}$ & $\begin{array}{c}\text { Sum of electronic } \\
\text { and thermal free } \\
\text { energies }(\mathrm{eV})\end{array}$ \\
\hline Dodecahedra & 0.0 & 0.0 & $\begin{array}{c}0.0 \\
13.54\end{array}$ & 0.0 & 0.0 & 0.0 & 0.0 \\
\hline LDL-1 & 5.24 & 4.66 & $\begin{array}{r}6.20 \\
39.15\end{array}$ & 1.17 & 1.19 & 1.42 & 0.98 \\
\hline LDL-2 & 5.27 & 4.58 & $\begin{array}{r}5.62 \\
26.74\end{array}$ & 1.28 & 1.20 & 1.50 & 0.93 \\
\hline HDL-1 & 5.60 & 5.05 & $\begin{array}{r}6.54 \\
26.04\end{array}$ & 1.28 & 1.19 & 1.51 & 0.99 \\
\hline HDL-2 & 5.55 & 5.18 & $\begin{array}{r}6.70 \\
38.91\end{array}$ & 0.74 & 0.78 & 0.98 & 0.65 \\
\hline Ice Ih & $\ldots$ & $\ldots$ & $\ldots$ & 3.83 & 2.14 & $(1.28)^{\mathrm{a}}$ & b \\
\hline
\end{tabular}

${ }^{a}$ Approximate due to imposed constraints.

${ }^{b}$ Not comparable due to imposed constraints. 
As is clear from Table I, there is a very large difference in gas phase stability between the dodecahedral cluster and the other optimized structures with the same number of molecules. For the wB97XD calculations, the alternative structures lie between 5.6 and $6.7 \mathrm{eV}$ higher in energy. TIP4P/2005 gives a similar picture with a span between 5.2 and $5.6 \mathrm{eV}$ and $\mathrm{MB}$-pol as well, but with somewhat smaller energy difference 4.6-5.2 eV. It is thus clear that the dodecahedral structures constitute low-energy configurations compared to those extracted from the simulations, but it has not been possible in the present work to explore all possible $\mathrm{H}$-bond arrangements to find global minima in each case. We note that already for a single dodecahedron of 20 molecules there are more than 32000 isomers in terms of $\mathrm{H}$-bond topology ${ }^{144}$ and these span a significant range in energy. 14

Characteristic for the dodecahedral cluster is that, compared to the alternative structures, the total dipole moment is quite small, 13.54 D (Table I), compared to 26-39 D for the other structures. The embedding in a polarizable medium thus brings down the energy differences significantly (Table I, column 4) to between 0.7 and $1.3 \mathrm{eV}$. Including also zero-point corrections makes no significant difference while including thermal corrections bring the energy differences down to between 0.6 and $1 \mathrm{eV}$. Interestingly, we find that also a cluster of 103 molecules cut out from hexagonal ice and energy-minimized at the same level of theory represents a higher energy than the fused dodecahedra (Table I). We emphasize that adding additional dodecahedra to make a third shell of dodecahedra introduces strain that hinders further growth of such structures in contrast to the growth of hexagonal templates. Per molecule, the energy differences between the dodecahedra and the other structures are very small, but for the agglomerate of 103 molecules it is significantly larger than $\mathrm{k}_{\mathrm{B}} \mathrm{T}$ at room temperature and might thus be significant and provide a kinetic hindrance to ice nucleation as pentagonal structures must be converted to hexagonal for unlimited growth in nucleation of ice. ${ }^{99}$ Our next step is then to investigate, using the TIP4P/2005 force-field as an approximation, for how long the dodecahedral clusters can survive in a solvation environment of equivalent molecules.

\section{Dodecahedra in simulated water}

In Fig. 8, the dodecahedral cluster solvated in TIP4P/2005 is shown at different times along the NVT simulation which was prepared as described in the section titled Methods. When the pressure of the system fluctuates, the average along the simulation is 26.8 bars, which may contribute to destroying the dodecahedral cluster. The ring statistics of the dodecahedral cluster and surrounding molecules are shown in Fig. 9, together with the bulk distribution at the same temperature. At the beginning of the simulation, the fraction of fiverings is much higher than the bulk, as expected, since the dodecahedral structure is still visible by visual inspection of the snapshot. The occurrence of five-ring members decreases with time, reaching the bulk value at about 20 ps. We can regard this time as the interval of time during which the dodecahedral seed cluster remains or the time required for this structural fluctuation to return to the average structure of water at that thermodynamic condition. At $30 \mathrm{ps,}$ the total distribution is close to that of the bulk simulation also in terms of the other ring-members, i.e., four- and six-membered rings.
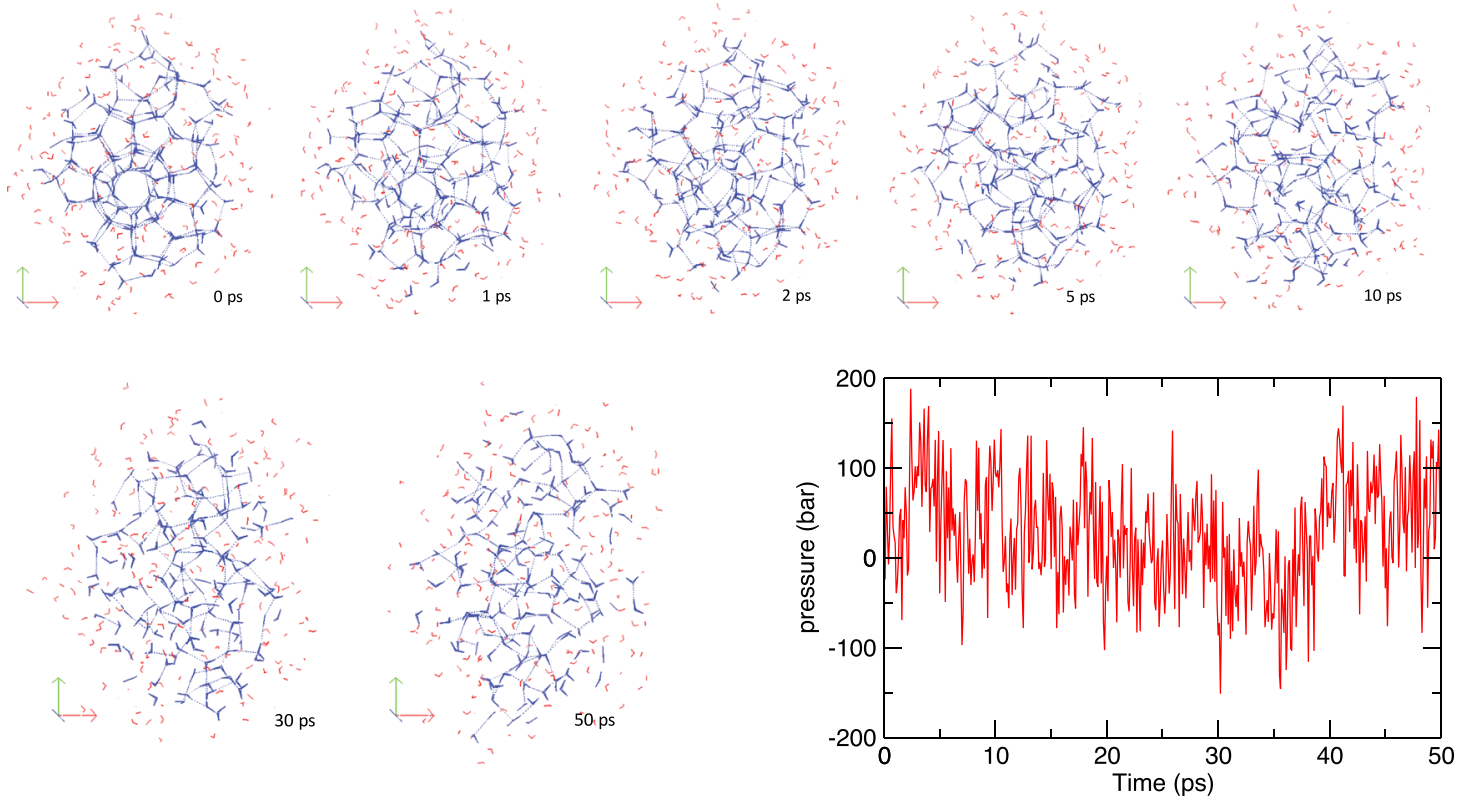

FIG. 8. Snapshots of the dodecahedra (blue) at different time-frames. HBs between molecules composing the dodecahedral cluster are shown as dotted lines. Solvent water molecules within $0.4 \mathrm{~nm}$ from the dodecahedra molecules are shown (red and white). Pressure along the NVT simulation of the solvated dodecahedra in water. 


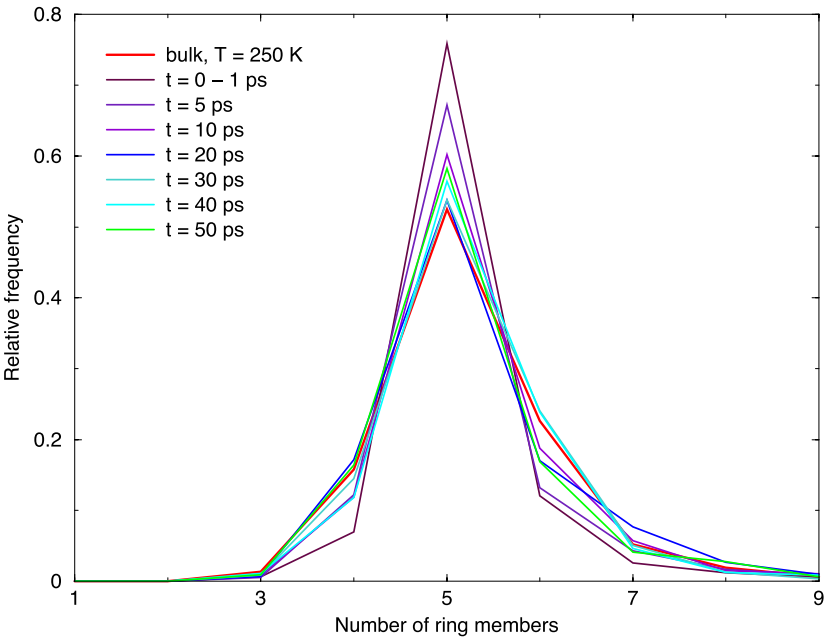

FIG. 9. Ring statistics within the volume seeded by a cluster of fused dodecahedra as a function of time compared to the average ring statistics of simulated TIP4P/2005 water (red).

\section{DISCUSSION AND CONCLUSIONS}

Clathratelike structures as tetrahedral templates have been suggested earlier, ${ }^{91-93,95,146,147}$ but abandoned since the resulting density was considered to become too low. In order to correct this, Pauling ${ }^{91}$ included a central molecule in the cavities and Yokoyama et al. ${ }^{147}$ added molecules to the facets, which resulted in the pentagonal facets converting to trimers. However, as can be expected of water in a picture of fluctuations between HDL and LDL, there are both a maximum and a minimum in the density. ${ }^{122,148}$ Considering both HDL and LDL to be normal, simple liquids for which the density increases continuously upon cooling, ${ }^{26}$ the density maximum is easily understood as the point where fluctuations into LDL-like structures become frequent enough to offset the density increase of the HDL component. Similarly, the density minimum results when the liquid has been converted to predominantly LDL, which then densifies upon further cooling.

The solvation of our proposed structure, the dodecahedral cluster, shows a stability around $20-30$ ps in the bulk during the MD simulations. There are several possible reasons for this limited lifetime. First, it could be that the proposed template, despite the very good agreement with both IR data and the O-O RDF at low temperature and the compatibility with the $\mathrm{x}$-ray spectroscopic data, in reality does not play a role in real water and the simulations actually reflect this fact.

On the other hand, even though experiments indicate the presence of specific, preferred structures, the time scale of the fluctuations between the two species in real water is at present not known, which makes it difficult to directly comment on the absolute value of the lifetime that we find. Density fluctuations in water on length-scales that depend on temperature have been reported based on small-angle x-ray scattering (SAXS) and assigned to structural fluctuations. . $^{65,90,122,149}$ Such fluctuations are discussed in a recent study using simulations of several supercooled and viscous liquids, ${ }^{150}$ where deviations from the expected Gaussian distribution were found in some cases for specific $\boldsymbol{k}$-vectors in the simulation, corresponding to symmetry on different length-scales. Such symmetries, or preferred local structures, have been discussed in the case of water or waterlike models in the vicinity of the glass transition by a number of authors. ${ }^{151-155}$

Assuming that the here discussed dodecahedral structures can be representative of LDL fluctuations in liquid water, they must still be considered to be extremely unlikely to appear through statistical fluctuations in MD simulations. This is due to the extremely large number of ways, $\sim 32000$ for a simple dodecahedron, ${ }^{144,156}$ to arrange the $\mathrm{H}$-bond network, where only a smaller fraction leads to favorable stabilization. Thus, in the present work, we have investigated seeding of the simulation with one such structure and we find remnants of the structure up to $\sim 30$ ps. However, there are many questions about our results. Had the seeded cluster been found to be stable over the length of the simulation, it would have been apparently more convincing, but still not a definite proof as the force-field might be considered to bias the simulation. Similarly, the limited stability of the template in the present investigation cannot be taken as proof that such templates can be excluded.

One concern here is the fluctuations in pressure in the simulations, which are due to the limited box size. These may artificially destabilize any specific structures, which in a simulation with smaller pressure fluctuations, would be found to be stable on a longer time scale. This, however, might require simulations with millions of molecules to alleviate pressure fluctuations in the equilibrated simulation box.

Another concern is whether the applied simulation model provides a balanced description between different structural species in simulated water. For instance, TIP4P/2005 is known to not reproduce the correct ratio between HDL and LDL structures at ambient conditions as defined by the LSI order parameter and lacks clear bimodality leading to a potential underestimation of LDL templates in the simulation which may influence the persistence of introduced templates; we note, however, a clear bimodality in the inherent structure (i.e., when quenched to $0 \mathrm{~K}$ ) using the same order parameter. $^{15}$

In the future, it would be worthwhile to adopt the same MDprotocol to study dodecahedral clusters also with potentials giving more or less structure, in order to see the effect of the model on the lifetimes of such templates. Another point connected to the density and the pressure is that, if in the simulation box there is an LDL structural fluctuation, there should also be HDL fluctuations such that the density of the system is the equilibrium density. A proposed structural fluctuation could thus in general not be stable inside the liquid, but it could be when more structural fluctuations are present, which, however, would necessitate significantly larger simulations with more reliable force-fields than have been possible for the present investigation.

The proposed dodecahedral templates for LDL fluctuations may seem contradicted by experience from traditional MD simulations and the present picture of water, but would be consistent with spectroscopic (IR, Raman, XAS, and XES) as well as scattering $\left[\mathrm{XRD}^{13,86,88}\right.$ and extended $\mathrm{X}$-ray-absorption fine structure (EXAFS ${ }^{158}$ ] data and would furthermore provide simple explanations of the enhanced solubility of nonpolar gases $\left(\mathrm{N}_{2}\right.$ and $\left.\mathrm{O}_{2}\right)$ below the compressibility minimum and the further increase around $20^{\circ} \mathrm{C}$ 
as low-density tetrahedral templates grow and can accommodate them.

\section{SUPPLEMENTARY MATERIAL}

See supplementary material for coordinates of the optimized structures and computed IR spectra of the additional HDL and LDL structures.

\section{ACKNOWLEDGMENTS}

This work was supported by the Swedish Research Council (VR) through Grant No. 2016-04875. Computational resources provided by the Swedish National Infrastructure for Computing (SNIC) at the HPC2N center are gratefully acknowledged.

\section{REFERENCES}

${ }^{1}$ M. F. Chaplin, Water Structure and Science, 2017, http://www.lsbu.ac.uk/water/ index.html.

${ }^{2}$ L. G. M. Pettersson, R. H. Henchman, and A. Nilsson, "Introduction: Water the most anomalous liquid,” Chem. Rev. 116, 7459-7461 (2016).

${ }^{3}$ P. Gallo, K. Amann-Winkel, C. A. Angell, M. A. Anisimov, F. Caupin, C. Chakravarty, T. Loerting, A. Z. Panagiotopoulos, J. Russo, H. Tanaka et al., "Water: A tale of two liquids," Chem. Rev. 116, 7463-7500 (2016).

${ }^{4}$ P. G. Debenedetti, “Supercooled and glassy water," J. Phys.: Condens. Matter 15, R1669-R1726 (2003).

${ }^{5}$ J. R. Errington and P. G. Debenedetti, "Relationship between structural order and the anomalies of liquid water," Nature 409, 318-321 (2001).

${ }^{6}$ T. Fransson, Y. Harada, N. Kosugi, N. A. Besley, B. Winter, J. Rehr, L. G. M. Pettersson, and A. Nilsson, "X-ray and electron spectroscopy of water," Chem. Rev. 116, 7551-7569 (2016).

${ }^{7}$ J. A. Sellberg, C. Huang, T. A. McQueen, N. D. Loh, H. Laksmono, D. Schlesinger, R. G. Sierra, D. Nordlund, C. Y. Hampton, D. Starodub et al., "Ultrafast X-ray probing of water structure below the homogeneous ice nucleation temperature," Nature 510, 381-384 (2014).

${ }^{8}$ K.-H. Kim, A. Späh, H. Pathak, F. Perakis, D. Mariedahl, K. Amann-Winkel, J. A. Sellberg, J. H. Lee, S. Kim, J. Park et al., "Maxima in the thermodynamic response and correlation functions of deeply supercooled water," Science 358, 1589-1593 (2017).

${ }^{9}$ A. Nilsson, S. Schreck, F. Perakis, and L. G. M. Pettersson, "Probing water with X-ray lasers," Adv. Phys.: X 1, 226-245 (2016).

${ }^{10}$ F. Perakis, G. Camisasca, T. J. Lane, A. Späh, K. T. Wikfeldt, J. A. Sellberg, F. Lehmkühler, H. Pathak, K.-H. Kim, K. Amann-Winkel et al., "Coherent X-rays reveal the influence of cage effects on ultrafast water dynamics," Nat. Commun. 9, 1917 (2018)

${ }^{11}$ G. A. Cisneros, K. T. Wikfeldt, L. Ojamäe, J. Lu, Y. Xu, H. Torabifard, A. P. Bartók, G. Csányi, V. Molinero, and F. Paesani, "Modeling molecular interactions in water: From pairwise to many-body potential energy functions," Chem. Rev. 116, 7501-7528 (2016).

${ }^{12}$ M. J. Gillan, D. Alfè, and A. Michaelides, "Perspective: How good is DFT for water?," J. Chem. Phys. 144, 130901 (2016).

${ }^{13}$ H. Pathak, A. Späh, K.-H. Kim, I. Tsironi, D. Mariedahl, M. Blanco, S. Huotari, V. Honkimäki, and A. Nilsson, J. Chem. Phys. 150, 224506 (2019).

${ }^{14} \mathrm{~J}$. Russo and H. Tanaka, “Understanding water's anomalies with locally favoured structures," Nat. Commun. 5, 3556 (2014).

${ }^{15}$ R. Shi, J. Russo, and H. Tanaka, "Common microscopic structural origin for water's thermodynamic and dynamic anomalies," J. Chem. Phys. 149, 224502 (2018).

${ }^{16}$ P. H. Poole, F. Sciortino, U. Essmann, and H. E. Stanley, "Phase-behavior of metastable water," Nature 360, 324-328 (1992).
${ }^{17}$ O. Mishima and H. E. Stanley, "The relationship between liquid, supercooled and glassy water," Nature 396, 329-335 (1998).

${ }^{18}$ O. Mishima, L. D. Calvert, and E. Whalley, "An apparently 1st-order transition between 2 amorphous phases of ice induced by pressure," Nature 314, 76-78 (1985).

${ }^{19} \mathrm{O}$. Mishima, "Reversible first-order transition between two $\mathrm{H}_{2} \mathrm{O}$ amorphs at $\sim 0.2 \mathrm{GPa}$ and $135 \mathrm{~K}$," J. Chem. Phys. 100, 5910-5912 (1994).

${ }^{20}$ S. Klotz, T. Strässle, R. J. Nelmes, J. S. Loveday, G. Hamel, G. Rousse, B. Canny, J. C. Chervin, and A. Saitta, "Nature of the polyamorphic transition in ice under pressure," Phys. Rev. Lett. 94, 025506 (2005).

${ }^{21}$ F. Perakis, K. Amann-Winkel, F. Lehmkühler, M. Sprung, D. Pettersson, J. A. Sellberg, H. Pathak, A. Späh, F. Cavalca, D. Schlesinger et al., "Diffusive dynamics during the high- to low-density transition in amorphous ices," Proc Natl. Acad. Sci. U. S. A. 114, 8193-8198 (2017).

${ }^{22}$ C. Lin, J. S. Smith, S. V. Sinogeikin, and G. Shen, "Experimental evidence of lowdensity liquid water upon rapid decompression," Proc. Natl. Acad. Sci. U. S. A. 115, 2010-2015 (2018).

${ }^{23}$ C. A. Angell, W. J. Sichina, and M. Oguni, "Heat capacity of water at extremes of supercooling and superheating," J. Phys. Chem. 86, 998-1002 (1982).

${ }^{24} \mathrm{H}$. Kanno and C. A. Angell, "Water: Anomalous compressibilities to $1.9 \mathrm{kbar}$ and correlation with supercooling limits," J. Chem. Phys. 70, 4008 (1979).

${ }^{25}$ R. J. Speedy and C. A. Angell, "Isothermal compressibility of supercooled water and evidence for a thermodynamic singularity at $-45^{\circ} \mathrm{C}$," J. Chem. Phys. 65, 851858 (1976).

${ }^{26}$ A. Nilsson and L. G. M. Pettersson, "The structural origin of anomalous properties of liquid water," Nat. Commun. 6, 8998 (2015).

${ }^{27}$ Y. Liu, J. C. Palmer, A. Z. Panagiotopoulos, and P. G. Debenedetti, "Liquidliquid transition in ST2 water," J. Chem. Phys. 137, 214505 (2012).

${ }^{28}$ J. C. Palmer, R. Car, and P. G. Debenedetti, "The liquid-liquid transition in supercooled ST2 water: A comparison between umbrella sampling and welltempered dynamics," Faraday Discuss. 167, 77-94 (2013).

${ }^{29}$ J. C. Palmer, F. Martelli, Y. Liu, R. Car, A. Z. Panagiotopoulos, and P. G. Debenedetti, "Metastable liquid-liquid transition in a molecular model of water," Nature 510, 385-388 (2014).

${ }^{30}$ J. C. Palmer, F. Martelli, Y. Liu, R. Car, A. Z. Panagiotopoulos, and P. G. Debenedetti, "Palmer et al. reply," Nature 531, E2-E3 (2016).

${ }^{31}$ T. A. Kesselring, G. Franzese, S. V. Buldyrev, H. J. Herrmann, and H. E. Stanley, "Nanoscale dynamics of phase flipping in water near its hypothesized liquid-liquid critical point," Sci. Rep. 2, 474 (2012).

${ }^{32}$ T. A. Kesselring, E. Lascaris, G. Franzese, and H. E. Stanley, "Finite-size scaling investigation of the liquid-liquid critical point in ST2 water and its stability with respect to crystallization," J. Chem. Phys. 138, 244506 (2013).

${ }^{33}$ D. T. Limmer and D. Chandler, "The putative liquid-liquid transition is a liquid-solid transition in atomistic models of water," J. Chem. Phys. 135, 134503 (2011).

${ }^{34}$ D. T. Limmer and D. Chandler, "The putative liquid-liquid transition is a liquidsolid transition in atomistic models of water. II," J. Chem. Phys. 138, 214504 (2013).

${ }^{35}$ J. C. Palmer, A. Haji-Akbari, R. S. Singh, F. Martelli, R. Car, A. Z. Panagiotopoulos, and P. G. Debenedetti, "Comment on 'The putative liquid-liquid transition is a liquid-solid transition in atomistic models of water' [I and II: J. Chem. Phys. 135, 134503 (2011); J. Chem. Phys. 138, 214504 (2013)],” J. Chem. Phys. 148, 137101 (2018).

${ }^{36}$ J. C. Palmer, P. H. Poole, F. Sciortino, and P. G. Debenedetti, "Advances in computational studies of the liquid-liquid transition in water and water-like models," Chem. Rev. 118, 9129-9151 (2018).

${ }^{37}$ J. W. Biddle, R. S. Singh, E. M. Sparano, F. Ricci, M. A. González, C. Valeriani, J. L. F. Abascal, P. G. Debenedetti, M. A. Anisimov, and F. Caupin, “Two-structure thermodynamics for the TIP4P/2005 model of water covering supercooled and deeply stretched regions,"J. Chem. Phys. 146, 034502 (2017).

${ }^{38}$ R. S. Singh, J. W. Biddle, P. G. Debenedetti, and M. A. Anisimov, "Twostate thermodynamics and the possibility of a liquid-liquid phase transition in supercooled TIP4P/2005 water,” J. Chem. Phys. 144, 144504 (2016)

${ }^{39} \mathrm{~V}$. Holten and M. A. Anisimov, "Entropy-driven liquid-liquid separation in supercooled water,” Sci. Rep. 2, 713 (2012) 
${ }^{40} \mathrm{Q}$. Sun, "Raman spectroscopic study of the effects of dissolved $\mathrm{NaCl}$ on water structure," Vib. Spectrosc. 62, 110-114 (2012).

${ }^{41} \mathrm{Q}$. Sun, "Local statistical interpretation for water structure," Chem. Phys. Lett. 568-569, 90-94 (2013).

${ }^{42}$ G. E. Walrafen, M. R. Fisher, M. S. Hokmabadi, and W.-H. Yang, "Temperature dependence of the low- and high-frequency Raman scattering from liquid water," J. Chem. Phys. 85, 6970-6982 (1986).

${ }^{43}$ G. E. Walrafen, M. S. Hokmabadi, and W.-H. Yang, "Raman isosbestic points from liquid water," J. Chem. Phys. 85, 6964-6969 (1986).

${ }^{44} \mathrm{P}$. L. Geissler, "Temperature dependence of inhomogeneous broadening: On the meaning of isosbestic points," J. Am. Chem. Soc. 127, 14930-14935 (2005).

${ }^{45}$ J. D. Smith, C. D. Cappa, K. R. Wilson, R. C. Cohen, P. L. Geissler, and R. J. Saykally, "Unified description of temperature-dependent hydrogen-bond rearrangements in liquid water," Proc. Natl. Acad. Sci. U. S. A. 102, 14171 (2005).

${ }^{46} \mathrm{Y}$. Maréchal, "The molecular structure of liquid water delivered by absorption spectroscopy in the whole IR region completed with thermodynamics data," J. Mol. Struct. 1004, 146-155 (2011).

${ }^{47}$ T. Morawietz, O. Marsalek, S. R. Pattenaudes, L. M. Streacker, D. Ben-Amotz, and T. E. Markland, "The interplay of structure and dynamics in the Raman spectrum of liquid water over the full frequency and temperature range," J. Phys. Chem. Lett. 9, 851-857 (2018).

${ }^{48}$ A. Taschin, P. Bartolini, R. Eramo, R. Righini, and R. Torre, "Evidence of two distinct local structures of water from ambient to supercooled conditions," Nat. Commun. 4, 2401 (2013).

${ }^{49}$ A. Nilsson, D. Nordlund, I. Waluyo, N. Huang, H. Ogasawara, S. Kaya, U. Bergmann, L.-Å. Näslund, H. Öström, P. Wernet et al., "X-ray absorption spectroscopy and X-ray Raman scattering of water; an experimental view," J. Electron Spectrosc. Relat. Phenom. 177, 99-129 (2010).

${ }^{50}$ D. Nordlund, H. Ogasawara, K. J. Andersson, M. Tatarkhanov, M. Salmerón, L. G. M. Pettersson, and A. Nilsson, "Sensitivity of X-ray absorption spectroscopy to hydrogen bond topology," Phys. Rev. B 80, 233404 (2009).

${ }^{51}$ J. A. Sellberg, S. Kaya, V. H. Segtnan, C. Chen, T. Tyliszczak, H. Ogasawara, D. Nordlund, L. G. M. Pettersson, and A. Nilsson, "Comparison of x-ray absorption spectra between water and ice: New ice data with low pre-edge absorption cross-section," J. Chem. Phys. 141, 034507 (2014).

${ }^{52}$ P. Wernet, D. Nordlund, U. Bergmann, M. Cavalleri, M. Odelius, H. Ogasawara, L. Å. Näslund, T. K. Hirsch, L. Ojamäe, P. Glatzel et al., "The structure of the first coordination shell in liquid water," Science 304, 995-999 (2004).

${ }^{53}$ J. Niskanen, C. J. Sahle, K. Gilmore, F. Uhlig, J. Smiatek, and A. Föhlisch, "Disentangling structural information from core-level excitation spectra," Phys. Rev. E 96, 013319 (2017).

${ }^{54}$ C. J. Sahle, C. Sternemann, C. Schmidt, S. Lehtola, S. Jahn, L. Simonelli, S. Huotari, M. Hakala, T. Pylkkänen, A. Nyrow et al., "Microscopic structure of water at elevated pressures and temperatures," Proc. Natl. Acad. Sci. U. S. A. 110, 6301-6306 (2013).

${ }^{55}$ S. Myneni, Y. Luo, L.-Å. Näslund, M. Cavalleri, L. Ojamäe, H. Ogasawara, A. Pelmenschikov, P. Wernet, P. Väterlein, C. Heske et al., "Spectroscopic probing of local hydrogen bonding structures in liquid water," J. Phys.: Condens. Matter 14, L213-L219 (2002).

${ }^{56}$ O. Fuchs, M. Zharnikov, L. Weinhardt, M. Blum, M. Weigand, Y. Zubavichus, M. Bär, F. Maier, J. D. Denlinger, C. Heske et al., "Isotope and temperature effects in liquid water probed by $\mathrm{x}$-ray absorption and resonant $\mathrm{x}$-ray emission spectroscopy," Phys. Rev. Lett. 100, 027801 (2008).

${ }^{57}$ L. Weinhardt, O. Fuchs, M. Blum, M. Bär, M. Weigand, J. D. Denlinger, Y. Zubavichus, M. Zharnikov, M. Grunze, C. Heske et al., "Resonant X-ray emission spectroscopy of liquid water: Novel instrumentation, high resolution, and the 'map' approach," J. Electron Spectrosc. Relat. Phenom. 177, 206-211 (2010).

${ }^{58}$ Y. Harada, J. Miyawaki, H. Niwa, K. Yamazoe, L. G. M. Pettersson, and A. Nilsson, "Probing the OH stretch in different local environments in liquid water," J. Phys. Chem. Lett. 8, 5487-5491 (2017).

${ }^{59}$ Y. Harada, T. Tokushima, Y. Horikawa, O. Takahashi, H. Niwa, M. Kobayashi, M. Oshima, Y. Senba, H. Ohashi, K. T. Wikfeldt et al., "Selective probing of the $\mathrm{OH}$ or OD stretch vibration in liquid water using resonant inelastic soft-X-ray scattering," Phys. Rev. Lett. 111, 193001 (2013).
${ }^{60}$ C. Huang, K. T. Wikfeldt, T. Tokushima, D. Nordlund, Y. Harada, U. Bergmann, M. Niebuhr, T. M. Weiss, Y. Horikawa, M. Leetmaa et al., "The inhomogeneous structure of water at ambient conditions," Proc. Natl. Acad. Sci. U. S. A. 106, 15214-15218 (2009).

${ }^{61}$ T. Tokushima, Y. Harada, Y. Horikawa, O. Takahashi, Y. Senba, H. Ohashi, L. G. M. Pettersson, A. Nilsson, and S. Shin, "High resolution X-ray emission spectroscopy of water and its assignment based on two structural motifs," J. Electron Spectrosc. Relat. Phenom. 177, 192-205 (2010).

${ }^{62}$ T. Tokushima, Y. Harada, O. Takahashi, Y. Senba, H. Ohashi, L. G. M. Pettersson, A. Nilsson, and S. Shin, "High resolution X-ray emission spectroscopy of liquid water: The observation of two structural motifs," Chem. Phys. Lett. 460, 387-400 (2008).

${ }^{63}$ T. Tokushima, Y. Horikawa, H. Arai, Y. Harada, O. Takahashi, L. G. M. Pettersson, A. Nilsson, and S. Shin, "Polarization dependent resonant $\mathrm{x}$-ray emission spectroscopy of $\mathrm{D}_{2} \mathrm{O}$ and $\mathrm{H}_{2} \mathrm{O}$ water: Assignment of the local molecular orbital symmetry," J. Chem. Phys. 136, 044517 (2012).

${ }^{64}$ J. Forsberg, J. Gråsjö, B. Brena, J. Nordgren, L. C. Duda, and J. E. Rubensson, "Angular anisotropy of resonant inelastic soft x-ray scattering from liquid water," Phys. Rev. B 79, 132203 (2009).

${ }^{65}$ J. H. Guo, Y. Luo, A. Augustsson, J. E. Rubensson, C. Såthe, H. Ågren, H. Siegbahn, and J. Nordgren, "X-ray emission spectroscopy of hydrogen bonding and electronic structure of liquid water," Phys. Rev. Lett. 89, 137402 (2002).

${ }^{66}$ S. Kashtanov, A. Augustsson, Y. Luo, J. H. Guo, C. Såthe, J. E. Rubensson, H. Siegbahn, J. Nordgren, and H. Ågren, "Local structures of liquid water studied by x-ray emission spectroscopy," Phys. Rev. B 69, 024201 (2004).

${ }^{67}$ K. M. Lange, R. Könecke, S. Ghadimi, R. Golnak, M. A. Soldatov, K. F. Hodeck, A. Soldatov, and E. F. Aziz, "High resolution X-ray emission spectroscopy of water and aqueous ions using the micro-jet technique," Chem. Phys. 377, 1-5 (2010).

${ }^{68}$ K. M. Lange, R. Könnecke, M. Soldatov, R. Golnak, J. E. Rubensson, A. Soldatov, and E. F. Aziz, "On the origin of the hydrogen-bond-network nature of water: X-ray absorption and emission spectra of water-acetonitrile mixtures," Angew. Chem. 123, 10809-10813 (2011).

${ }^{69}$ K. M. Lange, M. Soldatov, R. Golnak, M. Gotz, N. Engel, R. Könnecke, J.-E. Rubensson, and E. F. Aziz, "X-ray emission from pure and dilute $\mathrm{H}_{2} \mathrm{O}$ and $\mathrm{D}_{2} \mathrm{O}$ in a liquid microjet: Hydrogen bonds and nuclear dynamics," Phys. Rev. B 85, 155104 (2012).

${ }^{70}$ G. N. I. Clark, C. D. Cappa, J. D. Smith, R. J. Saykally, and T. Head-Gordon, "The structure of ambient water," Mol. Phys. 108, 1415-1433 (2010).

${ }^{71}$ J. D. Smith, C. D. Cappa, B. M. Messer, R. C. Cohen, and R. J. Saykally, "Response to 'Comment on 'Energetics of hydrogen bond network rearrangements in liquid water,"' Science 308, 793 b (2005).

${ }^{72}$ J. D. Smith, C. D. Cappa, K. R. Wilson, B. M. Messer, R. C. Cohen, and R. J. Saykally, "Energetics of hydrogen bond rearrangements in liquid water," Science 306, 851 (2004).

${ }^{73}$ O. Fuchs, M. Zharnikov, L. Weinhardt, M. Blum, M. Weigand, Y. Zubavichus, M. Bär, F. Maier, J. D. Denlinger, C. Heske et al., "Reply to 'Comment on 'Isotope and temperature effects in liquid water probed by X-ray absorption and resonant X-ray emission spectroscopy,"' Phys. Rev. Lett. 100, 249802 (2008).

${ }^{74}$ A. Nilsson, P. Wernet, D. Nordlund, U. Bergmann, M. Cavalleri, M. Odelius, H. Ogasawara, L.-Å. Näslund, T. K. Hirsch, L. Ojamäe et al., "Comment on 'Energetics of hydrogen bond network rearrangements in liquid water,"' Science $\mathbf{3 0 8}$, 793a (2005).

${ }^{75}$ L. G. M. Pettersson, T. Tokushima, Y. Harada, O. Takahashi, S. Shin, and A. Nilsson, "Comment on 'Isotope and temperature effects in liquid water probed by X-ray absorption and resonant X-ray emission spectroscopy,"” Phys. Rev. Lett. 100, 249801 (2008).

${ }^{76}$ G. N. I. Clark, G. Hura, J. Teixeira, A. K. Soper, and T. Head-Gordon, "Smallangle scattering and the structure of ambient liquid water," Proc. Natl. Acad. Sci. U. S. A. 107, 14003-14007 (2010).

${ }^{77}$ T. Head-Gordon and M. E. Johnson, "Tetrahedral structure or chains for liquid water,” Proc. Natl. Acad. Sci. U. S. A. 103, 7973-7977 (2006).

${ }^{78}$ T. Head-Gordon and M. E. Johnson, "Correction for Head-Gordon et al., Tetrahedral structure or chains for liquid water," Proc. Natl. Acad. Sci. U. S. A. 103, 16614 (2006). 
${ }^{79} \mathrm{~T}$. Head-Gordon and S. W. Rick, "Consequences of chain networks on thermodynamic, dielectric and structural properties for liquid water," Phys. Chem. Chem. Phys. 9, 83-91 (2007).

${ }^{80}$ A. K. Soper, J. Teixeira, and T. Head-Gordon, "Is ambient water inhomogeneous on the nanometer-length scale?," Proc. Natl. Acad. Sci. U. S. A. 107, E44 (2010).

${ }^{81}$ A. K. Soper, "Recent water myths," Pure Appl. Chem. 82, 1855-1867 (2010).

${ }^{82}$ J. Niskanen, M. Fondell, C. J. Sahle, S. Eckert, R. M. Jay, K. Gilmore, A. Pietzsch, M. Dantz, X. Lu, D. E. McNally et al., "Compatibility of quantitative X-ray spectroscopy with continuous distribution models of water at ambient conditions," Proc. Natl. Acad. Sci. U. S. A. 116, 4058-4063 (2019).

${ }^{83}$ L. G. M. Pettersson, Y. Harada, and A. Nilsson, "Do X-ray spectroscopies provide evidence for continuous distribution models of water at ambient conditions?," Proc. Natl. Acad. Sci. U. S. A. (submitted).

${ }^{84} \mathrm{~J}$. R. Scherer, M. K. Go, and S. Kint, "Raman spectra and structure of water from -10 to $90^{\circ}$," J. Phys. Chem. 78, 1304-1312 (1974).

${ }^{85}$ A. Nilsson, C. Huang, and L. G. M. Pettersson, "Fluctuations in ambient water," J. Mol. Liq. 176, 2-16 (2012).

${ }^{86}$ L. B. Skinner, C. J. Benmore, J. C. Neuefeind, and J. B. Parise, "The structure of water around the compressibility minimum," J. Chem. Phys. 141, 214507 (2014).

${ }^{87}$ L. B. Skinner, C. Huang, D. Schlesinger, L. G. M. Pettersson, A. Nilsson, and C. J. Benmore, "Benchmark oxygen-oxygen pair-distribution function of ambient water from $x$-ray diffraction measurements with a wide Q-range," J. Chem. Phys. 138, 074506 (2013).

${ }^{88}$ D. Schlesinger, K. T. Wikfeldt, L. B. Skinner, C. J. Benmore, A. Nilsson, and L. G. M. Pettersson, "The temperature dependence of intermediate range oxygenoxygen correlations in liquid water," J. Chem. Phys. 145, 084503 (2016).

${ }^{89}$ W. C. Röntgen, “Über die constitution des flüssigen Wassers," Ann. Phys. 281, 91-97 (1892).

${ }^{90}$ C. Huang, T. M. Weiss, D. Nordlund, K. T. Wikfeldt, L. G. M. Pettersson, and A. Nilsson, "Increasing correlation length in bulk supercooled $\mathrm{H}_{2} \mathrm{O}, \mathrm{D}_{2} \mathrm{O}$ and $\mathrm{NaCl}$ solution determined from small angle x-ray scattering," J. Chem. Phys. 133, 134504 (2010).

${ }^{91}$ L. Pauling, in Hydrogen Bonding, edited by D. Hadži (Pergamon Press, 1959), pp. 1-6.

${ }_{92}$ G. E. Walrafen, W.-H. Yang, and Y. C. Chu, "Raman evidence for the clathratelike structure of highly supercooled water," ACS Symp. Ser. 676, 287-308 (1997).

${ }^{93}$ H. Yokoyama, M. Kannami, and H. Kanno, "Existence of clathrate-like structures in supercooled water: X-ray diffraction evidence," Chem. Phys. Lett. 463, 99-102 (2008).

${ }^{94}$ J. L. F. Abascal and C. Vega, "A general purpose model for the condensed phases of water: TIP4P/2005," J. Chem. Phys. 123, 234505 (2005).

${ }^{95}$ R. J. Speedy, "Self-replicating structures in water," J. Phys. Chem. 88, 3364-3373 (1984).

${ }^{96}$ B. Santra, R. A. DiStasio, Jr., F. Martelli, and R. Car, "Local structure analysis in ab initio liquid water," Mol. Phys. 113, 2829-2841 (2015).

${ }^{97}$ M. Mandziuk, "From the trimer, through the pentamer, to liquid water," J. Mol. Struct. 1177, 168-176 (2019).

${ }^{98} \mathrm{~F}$. Martelli, "Unravelling the contribution of local structures to the anomalies of water: The synergistic action of several factors," J. Chem. Phys. 150, 094506 (2019).

${ }^{99} \mathrm{H}$. Shintani and H. Tanaka, "Frustration on the way to crystallization in glass," Nat. Phys. 2, 200-206 (2006).

${ }^{100} \mathrm{D}$. J. Anick, "Atypical water lattices and their possible relevance to the amorphous ices: A density functional study," AIP Adv. 3, 042119 (2013).

${ }^{101} \mathrm{E}$. Shiratani and M. Sasai, "Growth and collapse of structural patterns in the hydrogen bond network in liquid water," J. Chem. Phys. 104, 7671-7680 (1996).

${ }^{102}$ M. J. Frisch, G. W. Trucks, H. B. Schlegel, G. E. Scuseria, M. A. Robb, J. R. Cheeseman, G. Scalmani, V. Barone, G. A. Petersson, H. Nakatsuji et al., Gaussian 16, Revision B.01, Gaussian, Inc., Wallingford, CT, 2016.
${ }^{103}$ J. D. Chai and M. Head-Gordon, "Long-range corrected hybrid density functionals with damped atom-atom dispersion corrections," Phys. Chem. Chem. Phys. 10, 6615-6620 (2008).

${ }^{104}$ A. V. Marenich, C. J. Cramer, and D. G. Truhlar, "Universal solvation model based on solute electron density and a continuum model of the solvent defined by the bulk dielectric constant and atomic surface tensions," J. Phys. Chem. B 113, 6378-6396 (2009).

${ }^{105}$ P. Norman, D. M. Bishop, H. J. A. Jensen, and J. Oddershede, "Nearresonant absorption in the time-dependent self-consistent field and multiconfigurational self-consistent field approximations," J. Chem. Phys. 115, 10323-10334 (2001).

${ }^{106}$ P. Norman, D. M. Bishop, H. J. A. Jensen, and J. Oddershede, "Nonlinear response theory with relaxation: The first-order hyperpolarizability," J. Chem. Phys. 123, 194103 (2005).

${ }^{107}$ K. Aidas, C. Angeli, K. L. Bak, V. Bakken, R. Bast, L. Boman, O. Christiansen, R. Cimiraglia, S. Coriani, P. Dahle et al., "The Dalton quantum chemistry program system," Wiley Interdiscip. Rev.: Comput. Mol. Sci. 4, 269-284 (2014).

${ }^{108}$ T. Yanai, D. P. Tew, and N. C. Handy, "A new hybrid exchange-correlation functional using the coulomb-attenuating method," Chem. Phys. Lett. 393, 51-57 (2004).

${ }^{109}$ U. Ekström and P. Norman, "X-ray absorption spectra from the resonantconvergent first-order polarization propagator approach," Phys. Rev. A 74, 042722 (2006).

${ }^{110}$ U. Ekström, P. Norman, V. Carravetta, and H. Ågren, "Polarization propagator for X-ray spectra," Phys. Rev. Lett. 97, 143001 (2006).

${ }^{111}$ W. Kutzelnigg, U. Fleischer, and M. Schindler, NMR-Basic Principles and Progress (Springer Verlag, 1990).

${ }^{112}$ A. Bergner, M. Dolg, W. Kuechle, H. Stoll, and H. Preuß, "A $b$ initio energyadjusted pseudopotentials for elements of groups 13-17," Mol. Phys. 80, 14311441 (1993).

${ }^{113}$ N. Godbout, D. R. Salahub, J. Andzelm, and E. Wimmer, "Optimization of Gaussian-type basis sets for local spin density functional calculations. Part I. Boron through neon, optimization technique and validation," Can. J. Chem. 70, 560 (1992).

${ }^{114}$ T. Fransson, I. Zhovtobriukh, S. Coriani, K. T. Wikfeldt, P. Norman, and L. G. M. Pettersson, "Requirements on first-principles calculations of X-ray absorption spectra of liquid water," Phys. Chem. Chem. Phys. 18, 566-583 (2016).

${ }^{115} \mathrm{H}$. Ågren, V. Carravetta, O. Vahtras, and L. G. M. Pettersson, "Direct SCF direct static-exchange calculations of electronic spectra," Theor. Chem. Acc. 97, 14-40 (1997).

${ }^{116}$ M. E. Casida, in Recent Advances in Density Functional Methods, edited by D. P. Chong (World Scientific, Singapore, 1995), Vol. I.

${ }^{117}$ S. Hirata and M. Head-Gordon, "Time-dependent density functional theory within the Tamm-Dancoff approximation," Chem. Phys. Lett. 314, 291-299 (1999).

${ }^{118}$ Y. H. Shao, Z. T. Gan, E. Epifanovsky, A. T. B. Gilbert, M. Wormit, J. Kussmann, A. W. Lange, A. Behn, J. Deng, X. T. Feng et al., "Advances in molecular quantum chemistry contained in the Q-Chem 4 program package," Mol, Phys. 113, 184-215 (2015).

${ }^{119}$ J. D. Wadey and N. A. Besley, "Quantum chemical calculations of x-ray emission spectroscopy,” J. Chem. Theory Comput. 10, 4557-4564 (2014).

${ }^{120}$ W. J. Hehre, R. Ditchfield, and J. A. Pople, "Self-consistent molecular orbital methods. XII. Further extensions of Gaussian type basis sets for use in molecular orbital studies of organic molecules," J. Chem. Phys. 56, 2257-2261 (1972).

${ }^{121}$ D. van der Spoel, E. Lindahl, B. Hess, G. Groenhof, A. E. Mark, and H. J. C. Berendsen, "GROMACS: Fast, flexible, and free," J. Comput. Chem. 26, 1701-1718 (2005).

${ }^{122}$ K. T. Wikfeldt, C. Huang, A. Nilsson, and L. G. M. Pettersson, "Enhanced small-angle scattering connected to the Widom line in simulations of supercooled water," J. Chem. Phys. 134, 214506 (2011).

${ }^{123}$ G. Bussi, T. Zykova-Timan, and M. Parrinello, "Isothermal-isobaric molecular dynamics using stochastic velocity rescaling," J. Chem. Phys. 130, 074101 (2009). 
${ }^{124}$ H. J. C. Berendsen, J. P. M. Postma, W. F. van Gunsteren, A. DiNola, and J. R. Haak, "Molecular dynamics with coupling to an external bath," J. Chem. Phys. 81, 3684-3690 (1984).

${ }^{125}$ I. Zhovtobriukh, N. A. Besley, T. Fransson, A. Nilsson, and L. G. M. Pettersson, "Relationship between $\mathrm{x}$-ray emission and absorption spectroscopy and the local H-bond environment in water," J. Chem. Phys. 148, 144507 (2018).

${ }^{126}$ A. Nilsson and L. G. M. Pettersson, "Perspective on the structure of liquid water," Chem. Phys. 389, 1-34 (2011).

${ }^{127}$ H. Tanaka, "Simple physical model of liquid water," J. Chem. Phys. 112, 799809 (2000).

${ }^{128}$ G. R. Medders, V. Babin, and F. Paesani, "Development of a first principles water potential with flexible monomers. III. Liquid phase properties," J. Chem. Theory Comput. 10, 2906-2910 (2014).

${ }^{129}$ G. R. Medders, A. W. Götz, M. A. Morales, P. Bajaj, and F. Paesani, "On the representation of many-body interactions in water," J. Chem. Phys. 143, 104102 (2015).

${ }^{130}$ S. K. Reddy, D. R. Moberg, S. C. Straight, and F. Paesani, "Temperaturedependent vibrational spectra and structure of liquid water from classical and quantum simulations with the MB-pol potential energy function," J. Chem. Phys. 147, 244504 (2017).

${ }^{131}$ F. K. Gel'mukhanov, L. N. Mazalov, and A. V. Kondratenko, "A theory of vibrational structure in the X-ray spectra of molecules,” Chem. Phys. Lett. 46, 133 (1977).

${ }^{132}$ M. P. Ljungberg, L. G. M. Pettersson, and A. Nilsson, "Vibrational interference effects in $\mathrm{x}$-ray emission of a model water dimer: Implications for the interpretation of the liquid spectrum," J. Chem. Phys. 134, 044513 (2011).

${ }^{133}$ A. Nilsson, T. Tokushima, Y. Horikawa, Y. Harada, M. P. Ljungberg, S. Shin, and L. G. M. Pettersson, "Resonant inelastic $\mathrm{x}$-ray scattering of water," J. Electron Spectrosc. Relat. Phenom. 188, 84-100 (2013).

${ }^{134}$ M. Neeb, J. E. Rubensson, M. Biermann, and W. Eberhardt, "Coherent excitation of vibrational wave-functions observed in core hole decay spectra of $\mathrm{O}_{2}, \mathrm{~N}_{2}$ and CO," J. Electron Spectrosc. Relat. Phenom. 67, 261-274 (1994).

${ }^{135}$ M. P. Ljungberg, I. Zhovtobriukh, O. Takahashi, and L. G. M. Pettersson, "Core-hole-induced dynamical effects in the x-ray emission spectrum of liquid methanol," J. Chem. Phys. 146, 134506 (2017).

${ }^{136}$ O. Takahashi, M. P. Ljungberg, and L. G. M. Pettersson, "X-ray emission spectrum of liquid ethanol: Origin of split peaks," J. Phys. Chem. B 121, 11163-11168 (2017).

${ }^{137} \mathrm{M}$. Odelius, "Molecular dynamics simulations of fine structure in oxygen $\mathrm{K}$ edge x-ray emission spectra of liquid water and ice," Phys. Rev. B 79, 144204 (2009).

${ }^{138} \mathrm{M}$. Odelius, "Information content in $\mathrm{O}[1 \mathrm{~s}] \mathrm{K}$-edge X-ray emission spectroscopy of liquid water," J. Phys. Chem. A 113, 8176-8181 (2009).

${ }^{139}$ V. Vaz da Cruz, F. Gel'mukhanov, S. Eckert, M. Iannuzzi, E. Ertan, A. Pietzsch, R. C. Couto, J. Niskanen, M. Fondell, M. Dantz et al., "Probing hydrogen bond strength in liquid water by resonant inelastic X-ray scattering," Nat. Commun. 10, 1013 (2019).

${ }^{140}$ K. Yamazoe, J. Miyawaki, H. Niwa, A. Nilsson, and Y. Harada, "Measurements of ultrafast dissociation in resonant inelastic X-ray scattering of water," J. Chem. Phys. 150, 204201 (2019).

${ }^{141}$ M. Leetmaa, M. P. Ljungberg, A. P. Lyubartsev, A. Nilsson, and L. G. M. Pettersson, "Theoretical approximations to X-ray absorption spectroscopy of liquid water and ice," J. Electron Spectrosc. Relat. Phenom. 177, 135-157 (2010).
${ }^{142} \mathrm{~L}$. Kong, X. Wu, and R. Car, "Roles of quantum nuclei and inhomogeneous screening in the $\mathrm{x}$-ray absorption spectra of water and ice," Phys. Rev. B 86, 134203 (2012).

${ }^{143}$ M. Cavalleri, H. Ogasawara, L. G. M. Pettersson, and A. Nilsson, "The interpretation of $\mathrm{x}$-ray absorption spectra in water and ice," Chem. Phys. Lett. 364, 363 (2002).

${ }^{144}$ S. McDonald, L. Ojamäe, and S. J. Singer, "Graph theoretical generation and analysis of hydrogen-bonded structures with applications to the neutral and protonated water cube and dodecahedral clusters," J. Phys. Chem. A 102, 2824-2832 (1998).

${ }^{145} \mathrm{~A}$. Lenz and L. Ojamäe, "A theoretical study of water clusters: The relation between hydrogen-bond topology and interaction energy from quantum-chemical computations for clusters with up to 22 molecules," Phys. Chem. Chem. Phys. 7, 1905-1911 (2005).

${ }^{146}$ F. H. Stillinger, “Water revisited," Science 209, 451-457 (1980).

${ }^{147} \mathrm{H}$. Kanno, H. Yokoyama, and Y. Yoshimura, "A new interpretation of anomalous properties of water based on Stillinger's postulate," J. Phys. Chem. B 105, 2019-2026 (2001).

${ }^{148}$ D. Liu, Y. Zhang, C.-C. Chen, C.-Y. Mou, P. H. Poole, and S.-H. Chen, "Observation of the density minimum in deeply supercooled confined water," Proc. Natl. Acad. Sci. U. S. A. 104, 9570 (2007).

${ }^{149} \mathrm{C}$. Huang, K. T. Wikfeldt, T. Tokushima, D. Nordlund, Y. Harada, U. Bergmann, M. Niebuhr, T. M. Weiss, Y. Horikawa, M. Leetmaa et al., "Reply to Soper et al. "Fluctuations in water around a bimodal distribution of local hydrogen bonded structural motifs,"” Proc. Natl. Acad. Sci. U. S. A. 107, E45 (2010).

${ }^{150} \mathrm{U}$. R. Pedersen, "Statistics of small length scale density fluctuations in supercooled viscous liquids," J. Chem. Phys. 150, 094505 (2019).

${ }^{151}$ E. Donth, "The size of cooperatively rearranging regions at the glass transition," J. Non-Cryst. Solids 53, 325-330 (1982).

${ }^{152} \mathrm{~J}$. E. Hallett, F. Turci, and C. P. Royall, "Local structure in deeply supercooled liquids exhibits growing lengthscales and dynamical correlations," Nat. Commun. 9, 3272 (2018).

${ }^{153}$ C. P. Royall, R. W. Stephen, T. Ohtsuka, and H. Tanaka, "Direct observation of a local structural mechanism for dynamic arrest," Nat. Mater. 7, 556-561 (2008).

${ }^{154} \mathrm{H}$. Tanaka, "Bond orientational order in liquids: Towards a unified description of water-like anomalies, liquid-liquid transition, glass transition, and crystallization," Eur. Phys. J. E 35, 113 (2012).

${ }^{155}$ R. Pastore, A. Coniglio, and M. P. Ciamarra, "Dynamic phase coexistence in glass-forming liquids," Sci. Rep. 5, 11770 (2015)

${ }^{156}$ J. L. Kuo, J. V. Coe, S. J. Singer, Y. B. Band, and L. Ojamäe, "On the use of graph invariants for efficiently generating hydrogen bond topologies and predicting physical properties of water clusters and ice," J. Chem. Phys. 114, 2527-2540 (2001).

${ }^{157}$ K. T. Wikfeldt, A. Nilsson, and L. G. M. Pettersson, "Spatially inhomogeneous bimodal inherent structure in simulated liquid water," Phys. Chem. Chem. Phys, 13, 19918-19924 (2011).

${ }^{158}$ K. T. Wikfeldt, M. Leetmaa, A. Mace, A. Nilsson, and L. G. M. Pettersson, "Oxygen-oxygen correlations in liquid water; addressing the discrepancy between diffraction and EXAFS using a novel multiple-data set fitting technique," J. Chem. Phys. 132, 104513 (2010). 